\title{
Exogenous inter- $\alpha$ inhibitor proteins prevent cell death and improve ischemic stroke outcomes in mice
}

\author{
Louise D. McCullough, ${ }^{1}$ Meaghan Roy-0'Reilly, ${ }^{1}$ Yun-Ju Lai, ${ }^{1}$ Anthony Patrizz, ${ }^{1}$ Yan Xu, ${ }^{1}$ Juneyoung Lee, ${ }^{1}$ Aleah Holmes, ${ }^{1}$ \\ Daniel C. Kraushaar, ${ }^{2}$ Anjali Chauhan, ${ }^{1}$ Lauren H. Sansing, ${ }^{3}$ Barbara S. Stonestreet, ${ }^{4}$ Liang Zhu, ${ }^{5}$ Julia Kofler, ${ }^{6}$ \\ Yow-Pin Lim, ${ }^{7,8}$ and Venugopal Reddy Venna'
}

\begin{abstract}
'Department of Neurology, McCovern Medical School at University of Texas Health Science Center at Houston, Houston, Texas, USA. 2'Genomic and RNA Profiling Core, Baylor College of Medicine, Houston, Texas, USA. ${ }^{3}$ Department of Neurology, Yale University School of Medicine, New Haven, Connecticut, USA. ${ }^{4}$ Department of Pediatrics, Women and Infants Hospital of Rhode Island, The Alpert Medical School of Brown University, Providence, Rhode Island, USA. ${ }^{5}$ Biostatistics and Epidemiology Research Design Core, Center for Clinical and Translational Sciences, University of Texas Health Science Center at Houston, Houston, Texas, USA. ${ }^{6}$ Department of Pathology, University of Pittsburgh School of Medicine, Pittsburgh, Pennsylvania, USA. PProThera Biologics Inc., Providence, Rhode Island, USA. ${ }^{8}$ Department of Pathology and Laboratory Medicine, The Alpert Medical School of Brown University, Providence, Rhode Island, USA.
\end{abstract}

\begin{abstract}
Inter- $\alpha$ inhibitor proteins (IAIPs) are a family of endogenous plasma and extracellular matrix molecules. IAIPs suppress proinflammatory cytokines, limit excess complement activation, and bind extracellular histones to form IAIP-histone complexes, leading to neutralization of histone-associated cytotoxicity in models of sepsis. Many of these detrimental processes also play critical roles in the pathophysiology of ischemic stroke. In this study, we first assessed the clinical relevance of IAIPs in stroke and then tested the therapeutic efficacy of exogenous IAIPs in several experimental stroke models. IAIP levels were reduced in both ischemic stroke patients and in mice subjected to experimental ischemic stroke when compared with controls. Post-stroke administration of IAIP significantly improved stroke outcomes across multiple stroke models, even when given 6 hours after stroke onset. Importantly, the beneficial effects of delayed IAIP treatment were observed in both young and aged mice. Using targeted gene expression analysis, we identified a receptor for complement activation, C5aR1, that was highly suppressed in both the blood and brain of IAIP-treated animals. Subsequent experiments using C5aR1-knockout mice demonstrated that the beneficial effects of IAIPs are mediated in part by C5aR1. These results indicate that IAIP is a potential therapeutic candidate for the treatment of ischemic stroke.
\end{abstract}

\section{Introduction}

Stroke is now the primary cause of long-term adult disability and the fifth leading cause of mortality in the United States $(1,2)$. Ischemic strokes account for $80 \%-85 \%$ of all strokes (3). Despite the high burden of stroke, tissue plasminogen activator (t-PA) remains the only FDA-approved pharmacotherapy for ischemic stroke treatment. Unfortunately, t-PA can be used in only a small percentage of patients because of its short therapeutic time window and numerous contraindications (4-6). After the primary ischemic injury, an immediate cascade of damaging secondary pathways further exacerbates tissue damage, including the production of proinflammatory cytokines, the activation of destructive serine proteases, and the release of histones from dead and dying cells (7-10). A growing body of evidence suggests that dampening secondary damage after ischemic stroke is beneficial even if therapy is delayed beyond the window of t-PA treatment, as these secondary processes can persist for days to weeks after the initial injury $(11,12)$.

Conflict of interest: YPL and BSS hold a patent on inter- $\alpha$ inhibitor protein (IAIP) (US patent no. 9,572,87). Purified IAIP for these studies was provided by ProThera Biologics. YPL is cofounder of and holds significant financial interest in ProThera Biologics. Copyright: @ 2021, American Society for Clinical Investigation.

Submitted: October 9, 2020; Accepted: July 12, 2021; Published: September 1, 2021.

Reference information: J Clin Invest. 2021;131(17):e144898.

https://doi.org/10.1172/JCl144898.
Inter- $\alpha$ inhibitor proteins (IAIPs) are a family of structurally related proteins produced largely in the liver and found at high concentrations in the plasma (13-15). IAIPs have broad antiinflammatory activity, with the ability to inhibit destructive serine proteases, suppress proinflammatory cytokines $(16,17)$, augment antiinflammatory cytokine production, and reduce complement activation during systemic inflammatory challenges, including sepsis (18-20). In addition, IAIPs migrate into tissues during severe inflammation and stabilize the extracellular matrix, maintain neutrophils in a resting state, and reduce reactive oxygen species (ROS) production $(21,22)$.

Septic patients have been shown to have markedly decreased plasma levels of IAIPs and concomitant increases in IAIP-related fragments in the urine $(17,23,24)$, suggesting rapid consumption and clearance of IAIPs during systemic inflammatory challenges. Administration of exogenous IAIP reduces mortality in an animal model of sepsis, suggesting that IAIPs represent a powerful tool to prevent secondary damage caused by excessive inflammation (17). Similarly to sepsis, systemic inflammation is also present in stroke patients and is linked to poor outcome (25).

The major forms of IAIP found in plasma are $250-\mathrm{kDa}$ inter- $\alpha$ inhibitor (IaI), composed of 2 heavy chains ( $\mathrm{H} 1$ and $\mathrm{H} 2$ ) and a single light chain, and 125-kDa pre- $\alpha$ inhibitor (PaI), composed of 1 heavy chain (H3) and 1 light chain $(16,26)$. The light chain and the heavy chains are covalently linked by a glycosaminoglycan chain 
(15). The term "IAIP" refers to the preparation containing both major forms ( $I a I$ and $\mathrm{PaI}$ ) extracted from human plasma. The common light chain found in both IaI and PaI, termed "bikunin" (also known as urinary trypsin inhibitor or ulinastatin), possesses serine protease inhibitory activity (14). As indicated by its name, the initial studies following its discovery focused on the broad serine protease inhibitory activity exhibited by the light chain fragment of these complex proteins. The free light chain, or bikunin, has been shown to decrease infarct size and limit neuronal apoptosis in adult animals after cerebral ischemia $(27,28)$. However, due to high renal clearance, bikunin has a very short half-life (3-15 minutes) (29) when compared with the plasma-derived IAIP complexes (10-12 hours) (30). In addition, the role of the heavy chains in this protein family has been largely overlooked. Only later, when it was discovered that the heavy chain moieties were associated with hyaluronan, an important extracellular matrix component involved in physiological and pathological inflammatory processes, the biological functions of the heavy chain emerged. The heavy chains of IAIPs are involved in binding hyaluronan, complement proteins, coagulation factors, and extracellular histones both in the blood and in tissues $(16,19,22)$. Given the broad antiinflammatory effects and longer half-life of IAIP complexes, treatment with exogenous IAIP complexes may have greater beneficial therapeutic effects in adult ischemic stroke than bikunin alone.

We hypothesized that endogenous IAIPs would be rapidly consumed after an acute stroke as a result of the release of histones, excess complement activation, and significant inflammatory activation with ischemia, similar to sepsis. Furthermore, we hypothesized that the exogenous administration of purified IAIPs would improve outcomes after ischemic stroke, secondary to their capacity to neutralize histone cytotoxicity, decrease complement activation, and suppress detrimental proinflammatory signaling (22). To test this, we first used human plasma samples to determine whether IAIP and IAIP-histone complex levels were altered in stroke patients, and to determine whether changes in IAIP correlated with circulating cytokine levels. We then studied multiple mouse models of ischemic stroke, including transient, distal, permanent, and thromboembolic stroke in combination with t-PA. Next, to maximize the clinical relevance, we examined the efficacy of delayed IAIP administration on stroke outcome in aged animals, given the high prevalence of stroke in the aging population. To identify underlying mechanisms, we performed comprehensive multiplex gene expression analysis and used C5aR1 (also known as CD88) knockout mice to examine whether the therapeutic benefit of IAIPs is mediated in part by the complement system.

\section{Results}

Endogenous levels of IAIP are reduced acutely following ischemic stroke in mice and humans. In order to examine the effects of ischemic stroke on IAIP levels, ELISA was used to measure circulating IAIP and IAIP-histone complex levels at 24 hours after stroke onset both in the middle cerebral artery occlusion (MCAO) mouse model and in human ischemic stroke patients. IAIP levels were significantly decreased 24 hours after MCAO in mouse plasma $(P=0.0004$; Figure 1A). Conversely, IAIP-histone complexes were increased in stroke mice at 24 hours after stroke compared with sham control mice $(P=0.0466$; Figure $1 \mathrm{~B})$. To determine whether the pattern of IAIPs/IAIP-histone complexes seen in mice after stroke was clinically relevant, plasma levels of IAIP were measured in human patients 24 hours after stroke. In human patients, levels of IAIP were significantly lower 24 hours after ischemic stroke in comparison with non-stroke control patients $(P=0.0022$, effect size $=$ 0.63; Figure $1 C$ ). Ischemic stroke patients also showed a significant increase in circulating IAIP-histone complexes at 24 hours $(P=$ 0.0061 , effect size $=0.30$; Figure 1D), mirroring the findings seen in our mouse model. Linear regression analysis revealed that higher circulating IAIP levels were associated with lower levels of IFN- $\gamma$ $(P=0.0229$; Figure 1E), TNF- $\alpha(P=0.0397$; Figure $1 \mathrm{~F})$, and CCL11 $(P=0.018)$ in blood (Figure $1 G)$. These correlations remained significant even after adjustment for age and sex. Histological analysis of peri-infarct brain tissue revealed that IAIPs were expressed by multiple cell types in both mouse (Supplemental Figure 1; supplemental material available online with this article; https://doi. org/10.1172/JCI144898DS1) and human brain tissue, and that IAIP protein levels were significantly lower in post-stroke brains compared with controls ( $P=0.0003$; Figure $1, \mathrm{H}$ and I).

Immediate administration of IAIP reduces infarct volumes and limits edema after ischemic stroke. Mice were subjected to MCAO or sham surgery, with immediate intraperitoneal (i.p.) administration of $0,15,30$, or $45 \mathrm{mg} / \mathrm{kg}$ of exogenous IAIP (Figure 2A). Mice that received $30 \mathrm{mg} / \mathrm{kg}$ or $45 \mathrm{mg} / \mathrm{kg}$ IAIP were found to have significantly reduced infarct volumes compared with vehicle-treated animals 24 hours after stroke by 2,3,5-triphenyl-tetrazolium chloride (TTC) staining (compared with PBS, $P=0.0001$ in IAIP 30 $\mathrm{mg} / \mathrm{kg}$, and $P<0.0001$ in IAIP $45 \mathrm{mg} / \mathrm{kg}$; Figure 2B). Mice given a $30 \mathrm{mg} / \mathrm{kg}$ dose had significantly smaller ischemic injury at 24 hours by TTC staining (Figure 2, C and D), including reductions in cortical $(P<0.0001)$, striatal $(P=0.0004)$, and hemispheric injury $(P<0.0001)$. Mice that received IAIP treatment at the onset of stroke had significantly improved neurological deficit scores (NDSs) at 24 hours after stroke $(P=0.0057)$ compared with their vehicle-treated counterparts (Figure $2 \mathrm{E}$ ). Mice treated with IAIP had significantly lower plasma TNF- $\alpha$ levels $(P=0.0399)$, lower IL-6 levels $(P=0.0009)$, and higher IL-4 levels $(P<0.0001)$ compared with the vehicle group at 24 hours after stroke (Supplemental Figure 2, A-C). Brain edema was also significantly reduced in the ipsilateral hemisphere of IAIP-treated mice compared with vehicle-treated mice ( $P=0.0005$; Figure $2 \mathrm{~F})$. Circulating murine IAIP levels decreased rapidly after stroke and remained significantly decreased even 48 hours after stroke compared with the baseline values (Supplemental Figure 2D). Importantly, exogenously administered human IAIPs were detectable in the circulation 24 hours after the injection (Supplemental Figure 2E). No significant differences in physiological parameters were observed with IAIP treatment (Supplemental Table 1). In a separate cohort of mice, heat-inactivated IAIP (incubated at $56^{\circ} \mathrm{C}$ for 30 minutes) was tested using the same experimental design as an alternative control. In these experiments, mice treated with heat-inactivated IAIP developed significantly larger infarct volumes in the cortex $(P=0.0045)$, striatum $(P=0.0002)$, and total hemisphere $(P=$ 0.0057 ) in comparison with active IAIP (Supplemental Figure 3A).

Delayed administration of IAIP reduces infarct size and improves functional recovery after ischemic stroke. To determine whether the beneficial effects of IAIPs persisted in a delayed treatment paradigm 
A
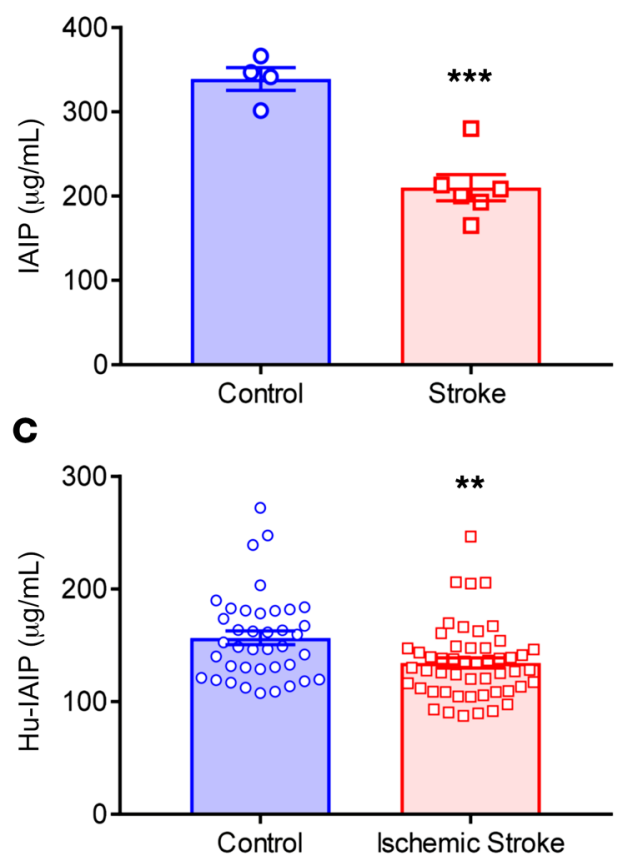

B

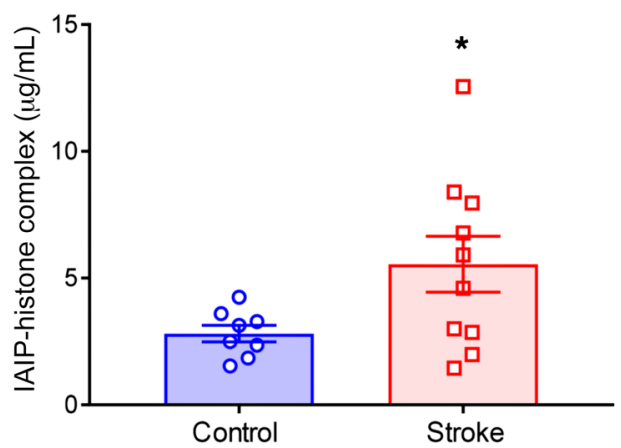

D

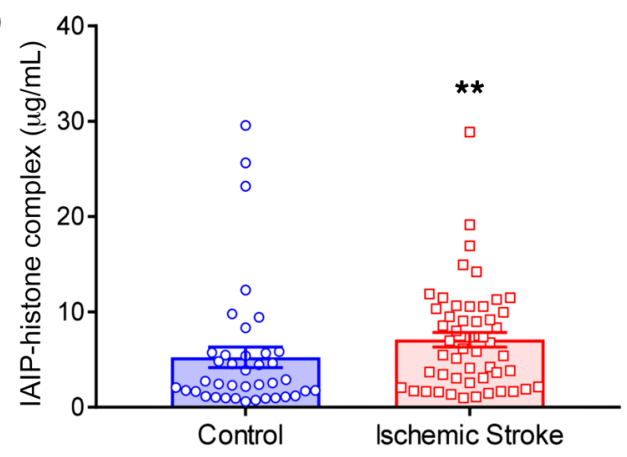

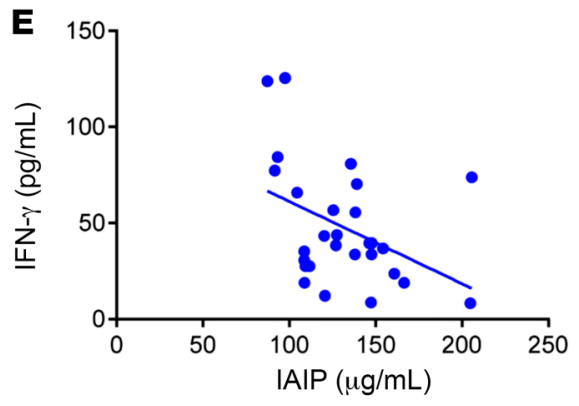

H
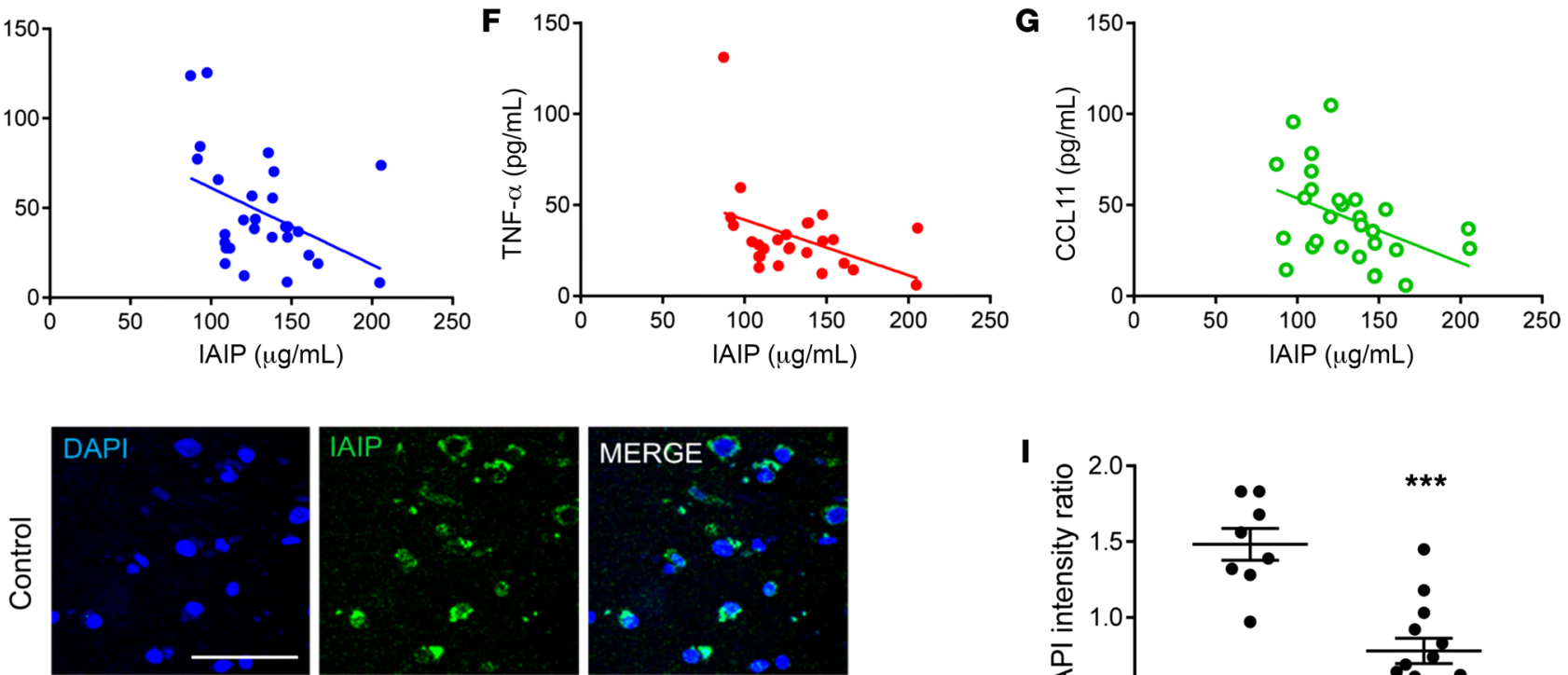
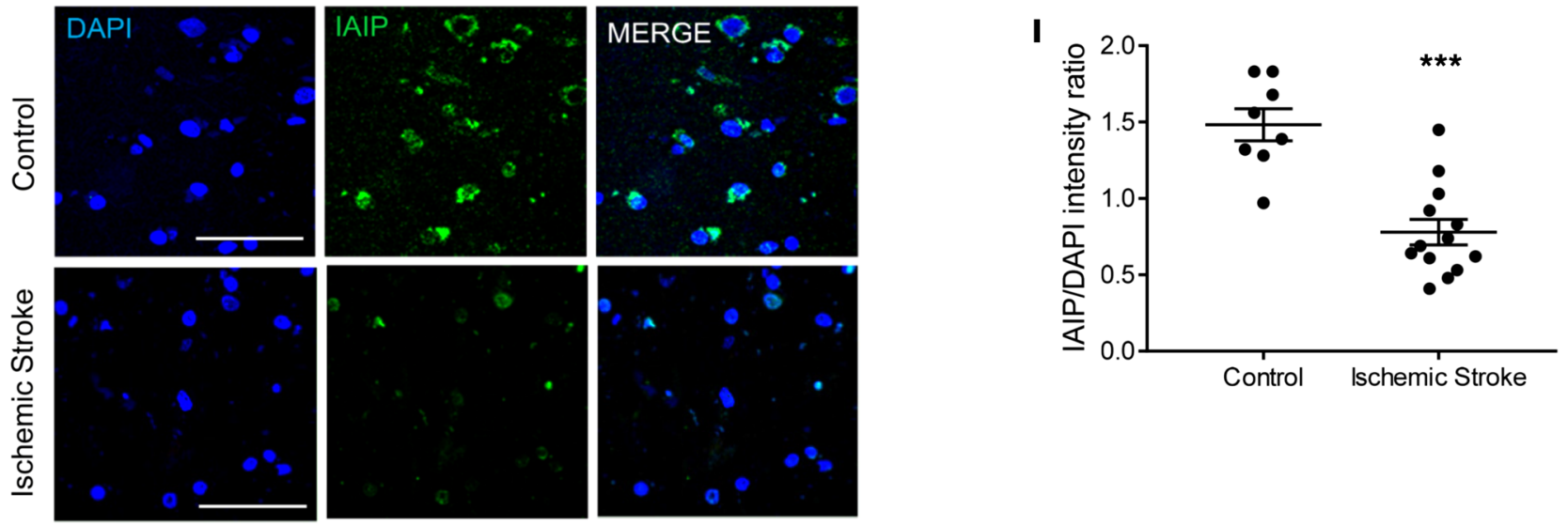

Figure 1. Endogenous IAIP levels are significantly reduced 24 hours after stroke; and IAIPs are expressed in both mouse and human brain. (A) Circulating IAIPs were significantly reduced in mouse plasma 24 hours after ischemic stroke compared with sham controls (data presented as mean \pm SEM; ${ }^{* * *} P=0.0004, t$ test). (B) IAIP-histone complexes were significantly elevated in mice at 24 hours after ischemic stroke compared with controls (mean \pm SEM; ${ }^{*} P=0.0466, t$ test). (C) Plasma IAIPs were significantly lower in ischemic stroke patients than in risk factor-matched controls 24 hours after stroke ( ${ }^{* *} P=0.0022$, Wilcoxon's rank sum test; effect size Cohen's $d=0.63$ ). An outlier test detected 1 outlier in the stroke group (ROUT method). After removal of the outlier, the $P$ value and effect size were 0.0011 and 0.73 , respectively. (D) IAIP-histone complexes were significantly higher in ischemic stroke patients than in control patients 24 hours after stroke ${ }^{* *} P=0.0061$, Wilcoxon's rank sum test; effect size Cohen's $d=0.30$ ). An outlier test detected 4 outliers in the control group and 1 in the stroke group. After removal of these outliers, $P$ was 0.0002 , and effect size $d$ was 0.93 . (E-C) Regression analysis revealed a significant negative correlation between inflammatory markers and plasma IAIP levels. Plasma from stroke patients with higher IAIP levels had lower TNF- $\alpha(P=0.0397)$, IFN- $\gamma(P=0.0229)$, and CCL11 $(P=0.018)$. (H) Immunohistochemistry on postmortem brains from stroke patients indicates that IAIPs are expressed in brain. Scale bars: $25 \mu \mathrm{m}$. (I) Fluorescence intensity data reveal that IAIP expression was significantly reduced in stroke brain (mean $\pm \mathrm{SEM} ;{ }^{* *} P=0.0003, t$ test). 


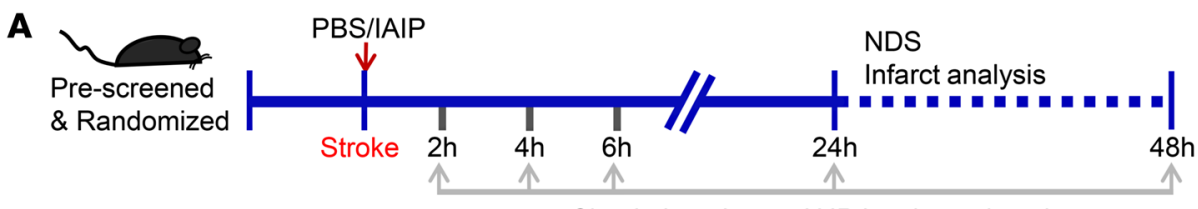

Circulating plasma IAIP levels analyzed
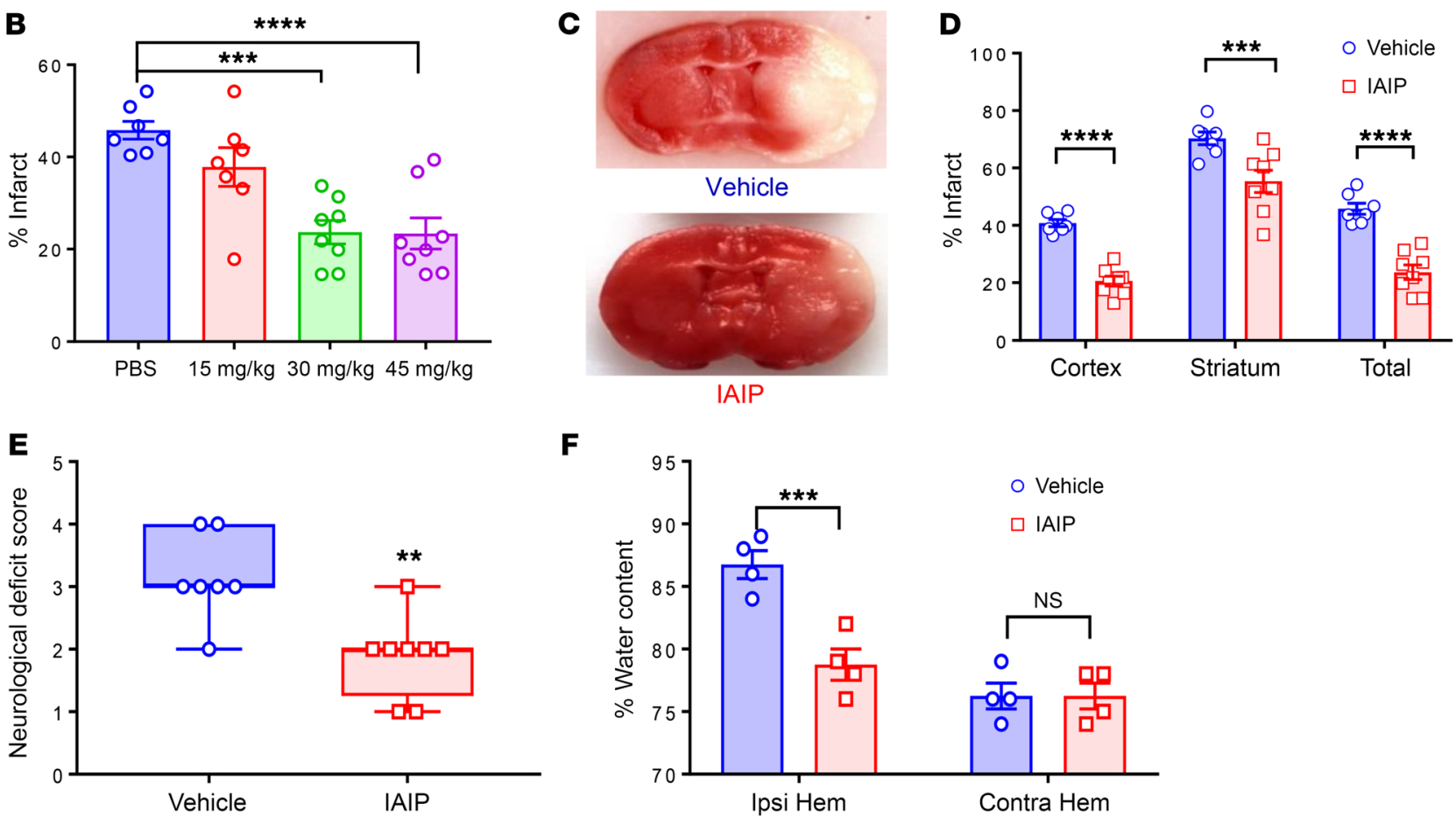

Figure 2. Immediate administration of exogenous IAIP reduces infarct size after MCAO in a dose-dependent manner. (A) The experimental timeline. (B) Total hemispheric infarct volume measurements were determined using TTC-stained brain sections. Significant protection is seen with IAIP treatment at doses of 30 and $45 \mathrm{mg} / \mathrm{kg}$ (PBS vs. IAIP $30 \mathrm{mg} / \mathrm{kg}$, ${ }^{* *} P=0.0001$; PBS vs. IAIP $45 \mathrm{mg} / \mathrm{kg}$, ${ }^{* * * *} P<0.0001 ; 1$-way ANOVA adjusted for multiple testing). (C) Representative TTC-stained images of vehicle-treated (control) and IAIP-treated (30 mg/kg) brains from young stroke mice ( 90 minutes MCAO, 24 hours reperfusion) treated immediately after stroke. (D) Quantification of infarct volumes shows a significant reduction in cortex, striatum, and total hemisphere at 24 hours after stroke (vehicle vs. IAIP $30 \mathrm{mg} / \mathrm{kg}$, mean $\pm \mathrm{SEM}$; ${ }^{* * *} P=0.0004,{ }^{* * * *} P<0.0001,2$-sample $t$ test adjusted for multiple testing). (E) IAIP treatment significantly reduced NDS compared with vehicle treatment. Data are presented as median and interquartile range $(* * P=0.0057$, Wilcoxon's rank sum test). (F) Percentage of water content in ipsilateral (Ipsi) and contralateral (Contra) hemispheres of young vehicle- and IAIP-treated mice $\left({ }^{* * *} P=\right.$ $0.0005, t$ test adjusted for multiple testing).

( $>4.5$ hours, the established window for t-PA), mice were subjected to MCAO and treated with either $30 \mathrm{mg} / \mathrm{kg}$ IAIP or vehicle at 6 hours with a repeated second dose at 18 hours after injury onset. A dose of $30 \mathrm{mg} / \mathrm{kg}$ was selected because prior experiments (Figure 2) found a similar neuroprotective benefit with both the $30 \mathrm{mg} / \mathrm{kg}$ and $45 \mathrm{mg} /$ $\mathrm{kg}$ doses. As IAIPs have a half-life of approximately 12 hours (30), we timed the second dose 12 hours after the initial dose as shown in the timeline in Figure 3A. Delayed treatment with IAIP at 6 hours with retreatment 18 hours after MCAO significantly reduced infarct volumes in the cortex $(P<0.0001)$, striatum $(P<0.0001)$, and total hemisphere $(P<0.0001)$ at 48 hours (Figure 3, B and C). We also tested the efficacy of IAIP when given by an i.v. injection via tail vein as an alternate route of administration. Mice that received a $30 \mathrm{mg} /$ $\mathrm{kg}$ dose had significantly smaller ischemic injury at 48 hours by TTC staining (Supplemental Figure 3B), including reductions in cortical cortex $(P=0.0107)$, striatum $(P=0.0063)$, and the total hemisphere $(P=0.0111)$. We then examined the effects of delayed IAIP dosing by administering the first dose at 6 hours and the second dose at 18 hours after MCAO on subacute infarct volume and behavioral recovery 7 days after stroke (cohort 2). Mice receiving delayed IAIP treatment had significantly smaller infarct volumes at 7 days after stroke compared with vehicle-treated controls in the cortex $(P<0.0001)$, striatum $(P<0.0001)$, and total hemisphere $(P<0.0001$; Figure $3, D$ and $\mathrm{E}$ ). In cohort 2, behavioral testing showed a significant beneficial effect of IAIP treatment on NDS $(P=0.0061$; Figure $3 \mathrm{~F})$ and rotational bias at 7 days after stroke $(P=0.037$; Figure $3 G)$.

Extended delayed IAIP treatment reduces brain atrophy and improves long-term recovery. Next, we performed 30-day survival experiments to determine the effects of IAIPs on long-term cerebral atrophy and functional outcomes. Mice were subjected to MCAO, then received $30 \mathrm{mg} / \mathrm{kg}$ IAIP i.p. starting at 6 hours with additional doses at 18 hours, day 2, day 3, day 5, day 7, and day 9 after stroke (Figure 4A). At the end of study follow-up (day 30), cerebral atrophy was significantly reduced in the IAIP-treated group $(P=0.0003$; Figure $4, B$ and $C$ ). There were no significant differences in cerebral blood flow at occlusion or at reperfusion between groups prior to initiation 
A

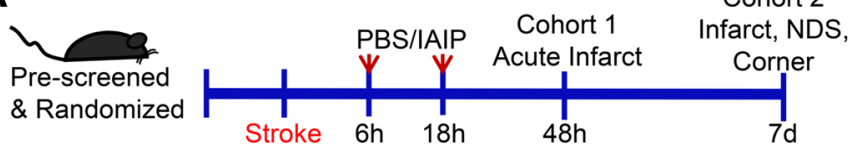

B

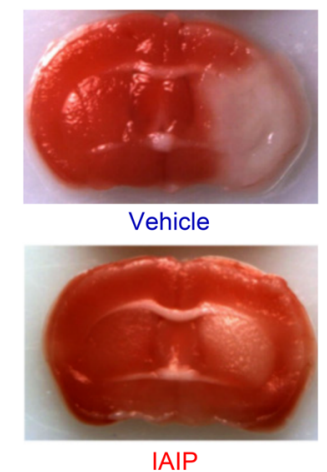

D
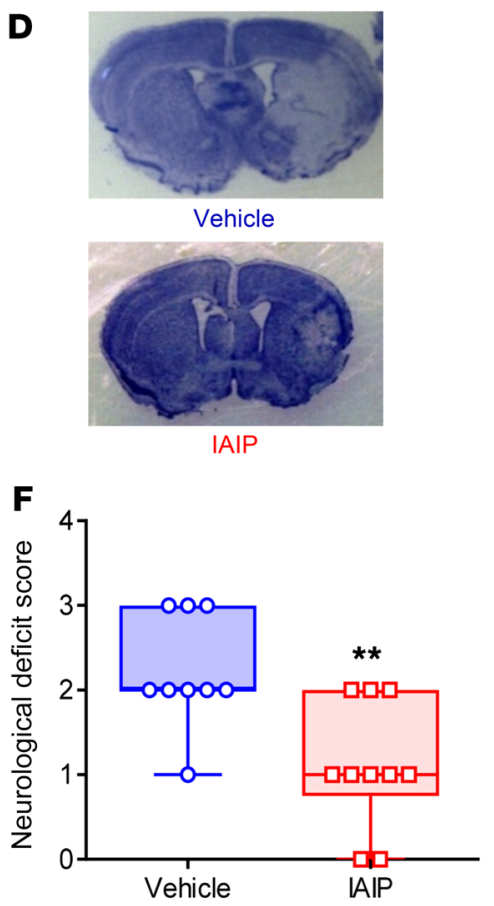
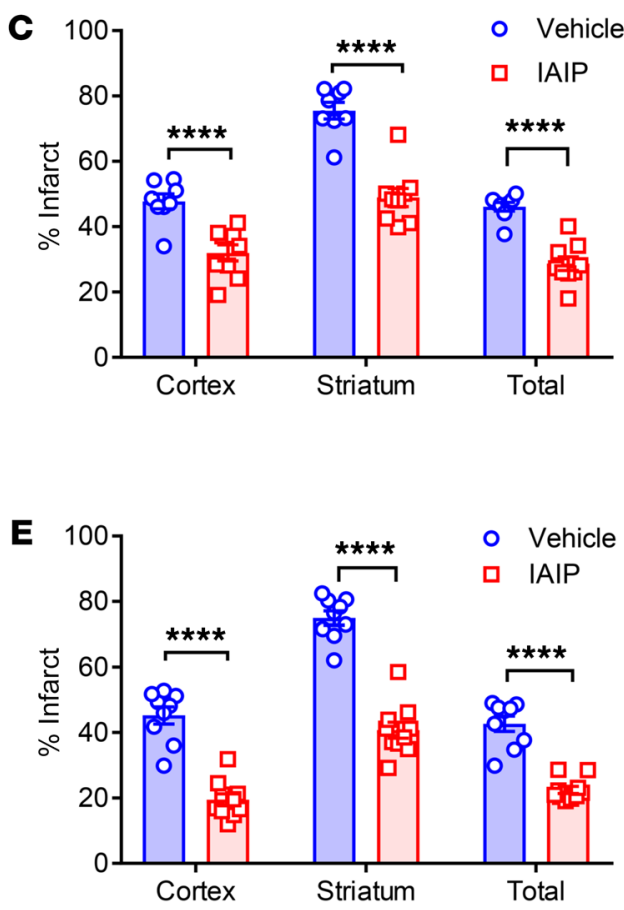

G

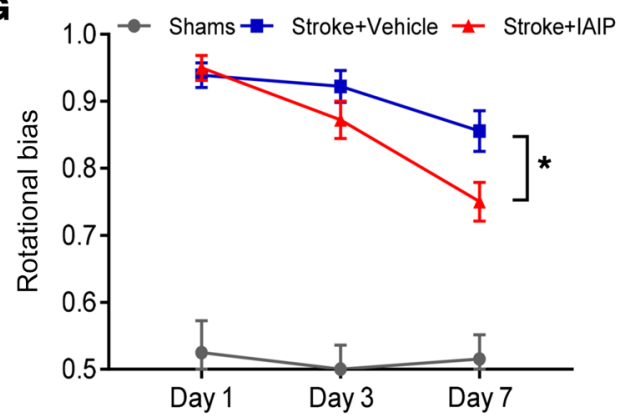

Figure 3. Delayed administration of IAIP 6 hours and 18 hours after MCAO reduces infarct volume and improves functional recovery. (A) Experimental timeline illustrates the delayed dosing regimen. (B and C) Representative TTC-stained coronal sections (vehicle and IAIP) (B) with quantification data (C). Delayed administration of $30 \mathrm{mg} / \mathrm{kg}$ IAIP at 6 hours and 18 hours after MCAO significantly reduced acute infarct volumes at 48 hours after stroke in the cortex, striatum, and total hemisphere ${ }^{* * * *} P<0.0001,2$-sample $t$ test adjusted for multiple testing). (D) In cohort 2 , representative cresyl violet-stained coronal section at day 7 after stroke (vehicle and IAIP). (E) Significant protection was seen with IAIP treatment in cohort 2 on day 7 after stroke in the cortex, striatum, and total hemispheres $\left({ }^{* * *} P\right.$ $<0.0001$ by 2 -sample, 2 -sided $t$ test adjusted for multiple testing). (F) IAIP treatment significantly improved functional outcomes in NDS at 48 hours $\left({ }^{* *} P=0.0061\right.$, Wilcoxon's rank sum test). (G) IAIP treatment significantly reduced rotational bias at day 7 after stroke by corner test $\left({ }^{*} P=0.0137\right.$, repeated-measures 2-way ANOVA adjusted for multiple testing). of treatment (Figure 4D). IAIP-treated mice had significantly lower mortality compared with the vehicle-treated group at day 30 after stroke with delayed treatment $(P=0.0347$; Figure 5A). IAIP-treated mice had significantly improved recovery in the corner test compared with the vehicle-treated group as measured by rotational bias on post-stroke day $7(P=0.049)$, day $14(P=0.0153)$, and day 21 $(P=0.0099$; Figure $5 \mathrm{~B})$. Delayed IAIP treatment reversed strokeinduced cognitive deficits as measured by the novel object recognition test (31), whereas the vehicle-treated stroke group showed significantly reduced preference for a novel object on post-stroke day 28 compared with the sham group $(P=0.017$; Figure $5 C)$. Mice that received post-stroke IAIP had improved learning ability (Figure $5 \mathrm{D}$ ) and memory retention in the Barnes maze test $(P<0.0001$; Figure 5 , $\mathrm{E}$ and $\mathrm{F})$. No significant differences in cognitive function were seen in sham groups treated with IAIP versus vehicle, which showed the expected preference for a novel versus a familiar object in this task.
Histological changes in the CA1 region of the hippocampus from the 30-day survival cohort were also examined, as these are hippocampus-dependent cognitive tasks $(32,33)$. Quantification of Nissl-stained sections of the CA1 region showed that stroke resulted in significant neuronal loss in the CA1 region in comparison with vehicle-treated animals ( $P=0.0009$; Figure 5 , $\mathrm{G}$ and $\mathrm{H})$. IAIP-treated stroke animals had significantly reduced neuronal loss compared with vehicle-treated stroke animals $(P=0.0316)$, but no significant difference between sham groups was noted (Figure $5 \mathrm{H}$ ).

Delayed IAIP treatment improves chronic functional recovery in aged mice after distal stroke. Next, we used a mild model of stroke (distal MCAO occlusion; ref. 34) to ensure that the observed beneficial effects of exogenous IAIP treatment in aged mice were not simply due to improved survival. Aged male mice (18-20 months) were subjected to distal stroke and treated with delayed IAIP or vehicle starting at 6 hours after stroke, with additional 
A

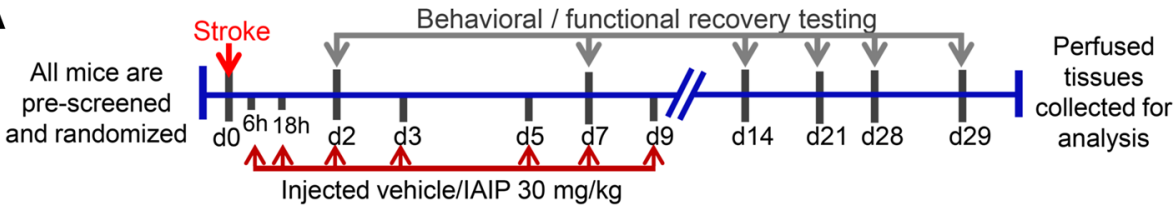

B

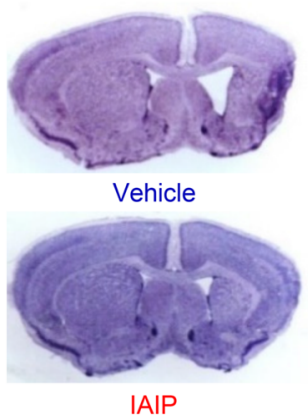

C

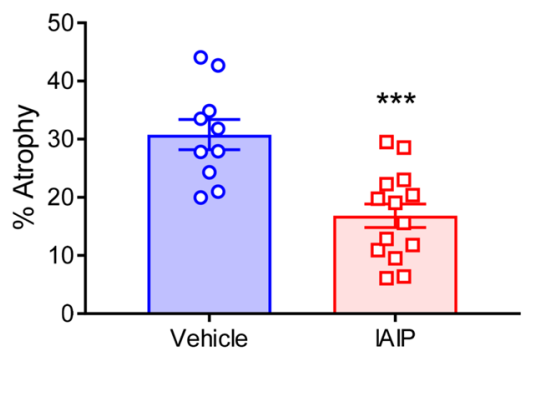

D

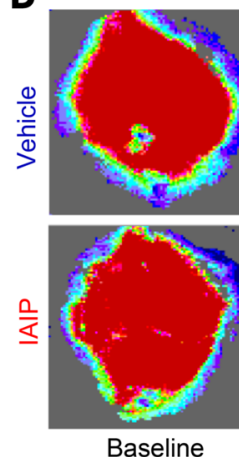

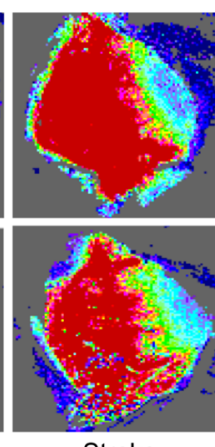

Stroke

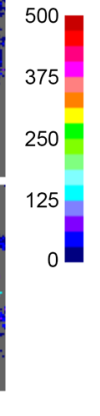

Figure 4. Extended delayed IAIP treatment reduces brain atrophy. (A) Young male mice were randomized and subjected to stroke or sham surgery (60 minutes MCAO) followed by treatment with IAIP or vehicle and behavioral testing out to post-stroke day 30 . (B) Representative images of atrophy from vehicle- and IAIP-treated groups after stroke. (C) Quantification of brain atrophy in vehicle- and IAIP-treated mice demonstrated a significant reduction in atrophy in IAIP-treated mice $\left({ }^{* *} P=0.0003, t\right.$ test). (D) Both IAIP- and vehicle-treated groups had equivalent decreases in blood flow at occlusion and reperfusion, as measured by laser speckle.

doses given at 18 hours, day 2, day 3, day 5, day 7, and day 9 after stroke onset. Aged mice treated with IAIP had no significant difference in mortality compared with the vehicle-treated group (1 mouse died from each group during follow-up). Quantification of brain atrophy revealed significantly less hemispheric atrophy with IAIP treatment compared with vehicle at day 30 after distal stroke in aged male mice $(7.33 \% \pm 1.49 \%$ in vehicle vs. $3.48 \% \pm$ $0.63 \%$ in IAIP-treated mice; $P=0.0207$; data not shown). Mice treated with IAIP had significantly reduced latency to remove tape in an adhesive-tape removal test (35) compared with the vehicle group at day $7(P=0.0032)$, day $14(P=0.0002)$, and day $21(P=0.0344)$ with repeated-measures ANOVA adjusted for multiple testing (Figure 5I).

IAIP treatment is protective in both the permanent occlusion model and the thromboembolic model when given in combination with $t-P A$. To increase the likelihood of clinical translation, it is important to test the efficacy of potential novel treatments in multiple experimental stroke models. To that end, mice were subjected to the permanent occlusive stroke model (pMCAO) without reperfusion, and infarct size was assessed 48 hours after stroke (Figure 6A). Mice receiving IAIP after permanent ischemic occlusion showed significantly smaller infarcts than vehicle-treated mice (total infarct: $P=$ 0.0111; cortex: $P=0.0185$; striatum: $P=0.0054$ ) (Figure 6B). There was no significant difference in NDSs after pMCAO in mice treated with IAIP $(P=0.0706)$ at 24 hours after stroke (Figure 6C). Howev$\mathrm{er}$, in the hang wire test, in which the latency to fall is measured as an indication of grip strength (36), mice receiving IAIP held on to the wire for significantly longer than the vehicle-treated group 48 hours after stroke ( $P=0.0378$; Figure 6D).

It is important to assess the impact of combined IAIP/t-PA therapy on ischemic stroke outcome because t-PA therapy is currently the pharmacological gold standard to treat ischemic stroke. Therefore, we used a thromboembolic model of experimental ischemic stroke (37) followed by a 30-minute continuous t-PA reperfusion starting 30 minutes after occlusion (Figure 6E). Mice treated with IAIP in combination with t-PA had smaller infarcts compared with mice treated with t-PA alone after thromboembolic stroke, with significant reductions seen in the total hemispheric infarct $(P=0.0444$; Figure $6 \mathrm{~F})$. Mice treated with IAIP had a significantly lower NDS $(P=0.046$; Figure $6 G)$ at 24 hours and significantly better grip strength as measured by the hang wire test compared with the vehicle-treated group $(P=0.0015)$ at 48 hours after stroke (Figure 6H).

Mice treated with IAIP also had a reduced amount of poststroke hemorrhagic transformation, as quantified by hemoglobin concentration from the ipsilateral brain hemisphere $(P=0.0487$; Figure 6I). To further confirm whether the reduced hemoglobin in the IAIP-treated group was a result of reduced blood-brain barrier (BBB) leakage, we performed immunohistochemistry on brain sections collected at 48 hours after reperfusion. There was a significant reduction in the fibrinogen-positive area in IAIP-treated mice in the ipsilateral hemisphere. Mice treated with t-PA plus IAIP also had significantly lower brain hemoglobin concentrations compared with vehicle-treated mice $(P=0.0196$; Figure 6, $\mathrm{J}$ and $\mathrm{K}$ ). A tail bleed assay (38) was used to determine whether IAIP contributed to differences in coagulation time. There were no significant differences in coagulation between IAIP- and vehicle-treated groups 5 hours after t-PA administration (mean \pm SEM; $P=0.7831$; Supplemental Figure 4).

Delayed IAIP treatment reduces infarct size, decreases mortality, and improves functional outcome in aged mice after stroke. Next, we tested whether exogenous IAIP treatment was beneficial in aged subjects, as ischemic stroke is largely a disease of aging. Aged male mice (18-20 months) were subjected to MCAO and treated with delayed IAIP or vehicle at 6 hours, 18 hours, day 2, and day 3 after stroke onset. Aged mice receiving IAIP had significantly reduced 
A

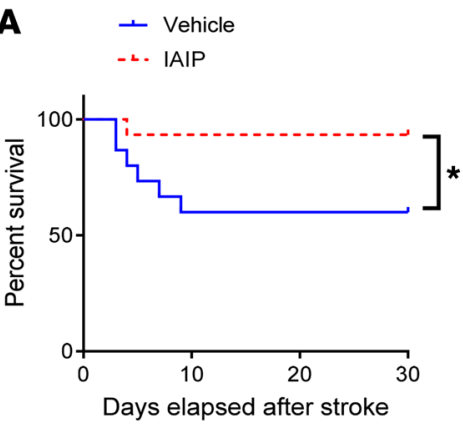

D

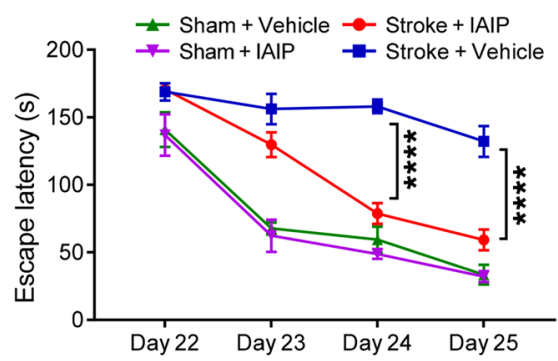

G
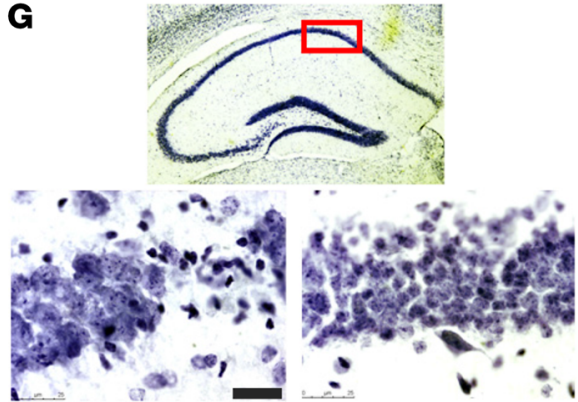

Stroke + Vehicle
Stroke + IAIP
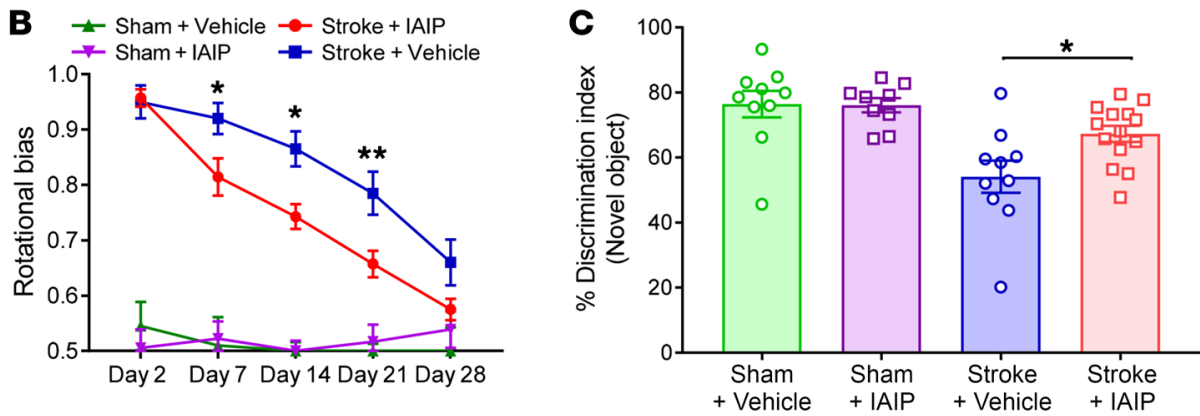

E

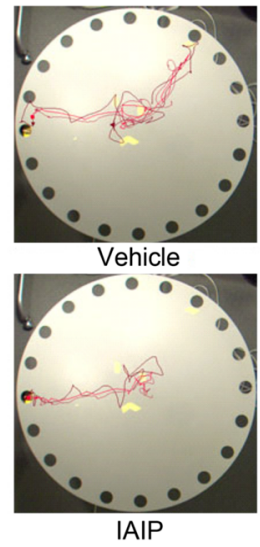

H

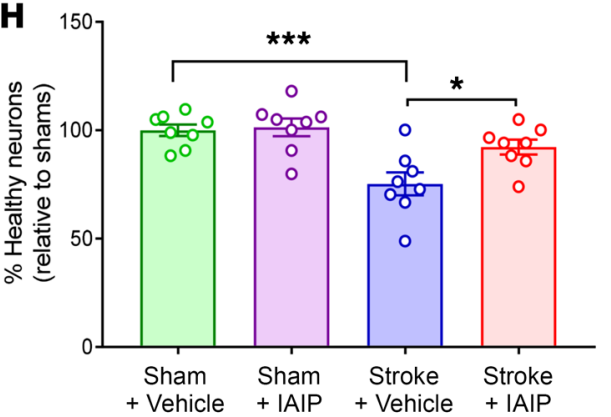

$\mathbf{F}$

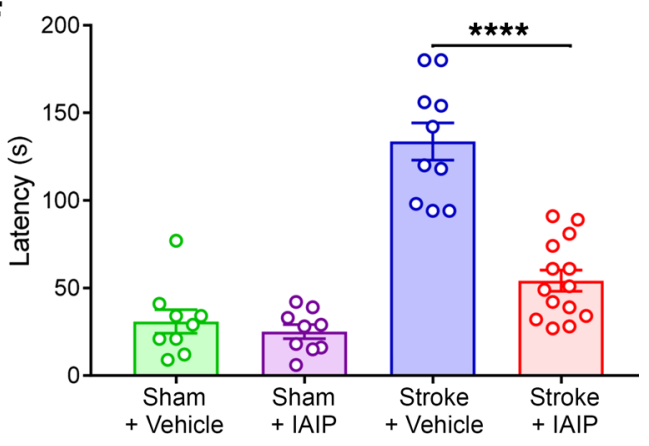

Figure 5. Delayed exogenous IAIP treatment reduces post-stroke mortality and improves long-term recovery. (A) IAIP-treated animals had improved mortality rates compared with vehicle-treated animals by day 15; no additional animals died between days 15 and 30 ( ${ }^{*} P=0.0347$, log-rank test). (B) IAIP treatment significantly reduced rotational bias in the corner test versus vehicle at day $7\left({ }^{*} P=0.0491\right)$, day $14\left({ }^{*} P=0.0153\right)$, and day $21\left({ }^{* *} P=0.0099\right)$ (repeated-measures ANOVA adjusted for multiple testing). IAIP-treated mice recovered by post-stroke day 28 compared with shams; vehicle-treated mice remained significantly impaired compared with shams $(P=0.0074)$. (C) IAIP treatment significantly improved stroke-induced cognitive deficits as measured by the novel object recognition test in young male mice at post-stroke day $28\left({ }^{*} P=0.041\right.$, 2-way ANOVA). (D) Mice treated with IAIP had significantly better learning ability compared with the vehicle group during training sessions from day 22 to day 25 (day $23,{ }^{* * *} P<0.0001$; day 25 , ${ }^{* * *} P<0.0001$; repeated-measures 2-way ANOVA adjusted for multiple testing). (E) Representative images of Barnes maze mouse movement tracking. (F) Stroke mice treated with IAIP had improved memory retention at day 27 compared with the vehicle group ( ${ }^{* * *} P<0.0001,2$-way ANOVA), with no significant difference in sham groups. (C) Representative Nissl-stained sections of CA1 region of hippocampus. Scale bar: $25 \mu \mathrm{m}$. (H) Quantification of neuronal counts demonstrated significant neuronal loss after stroke $\left({ }^{* *} P=0.0009\right)$; IAIP treatment significantly reduced neuronal loss $\left({ }^{*} P=0.0316\right)$ compared with vehicle, normalized and presented relative to sham, by 2-way ANOVA adjusted for multiple testing. (I) IAIP significantly improved recovery after distal stroke in aged male mice versus vehicle at day $7\left({ }^{* *} P=0.0032\right)$, day $14\left({ }^{* *} P=0.0002\right)$, and day $21\left({ }^{*} P=0.0344\right)$ (repeated-measures ANOVA adjusted for multiple testing).

infarct volumes compared with those receiving vehicle, including cortical $(P=0.0020)$, striatal $(P<0.0001)$, and total hemispheric infarcts $(P=0.0113)$, when analyzed at post-stroke day 7 (Figure 7 , $A$ and B). Aged mice treated with IAIP also had significantly lower mortality compared with the vehicle-treated group at post-stroke day 7 of reperfusion $(P=0.0337$; Figure $7 C)$.

In addition to reduced infarct size, IAIP-treated mice also had significantly improved recovery in spontaneous locomotor activity by post-stroke day $7(P=0.0323)$, as measured by total beam breaks (Figure 7D). Aged male mice treated with delayed IAIP showed significantly improved NDSs over time and improved significantly by post-stroke day 7 compared with vehicle-treated mice (Figure 7E). Aged animals that received IAIP had restored gait symmetry by day 7 in DigiGait analysis (Mouse Specifics Inc.) (39), indicating an improved return to baseline limb function compared with animals treated with vehicle after stroke $(P=0.0147$; Figure $7 F)$. Data from flow cytometry revealed that IAIP-treated animals had significantly reduced stroke-induced neutrophil infiltration to ipsilateral hemisphere at 3 days after stroke compared with the vehicle group $(P=0.0105$; Figure $7 G)$. 
A
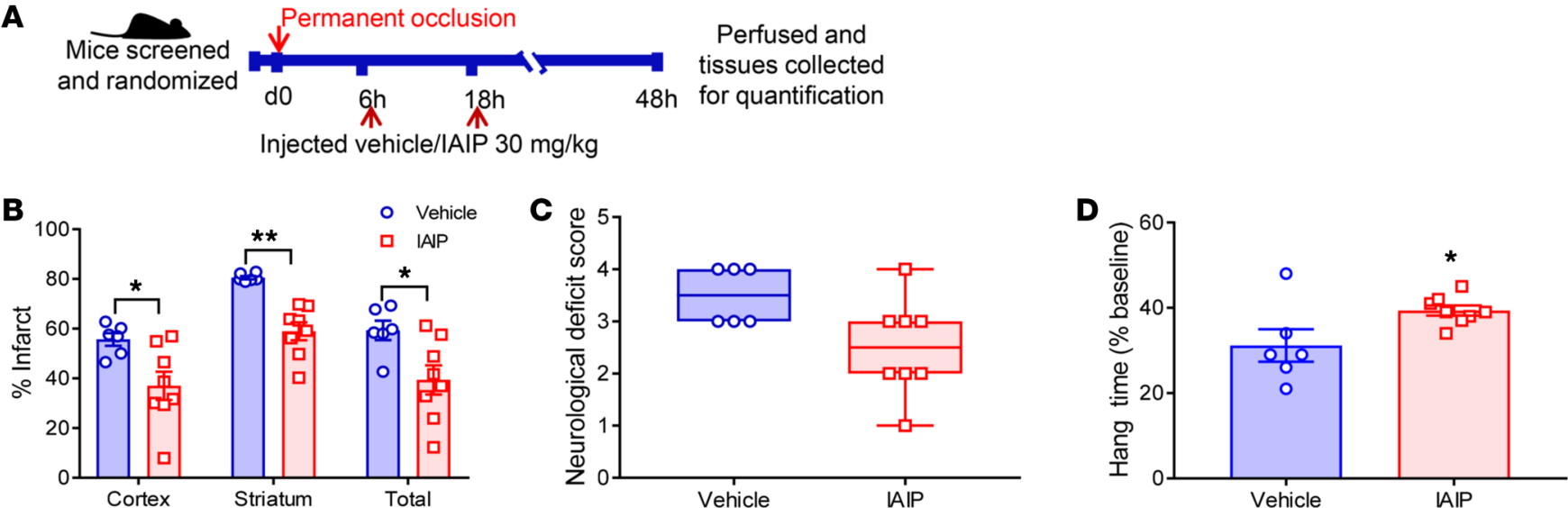

E

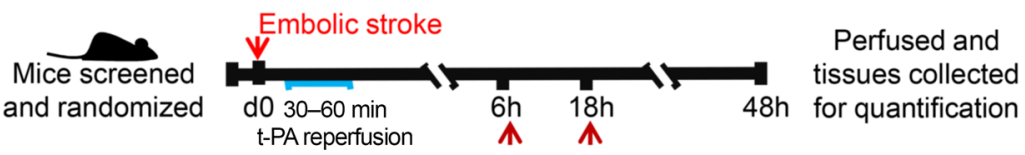

Injected vehicle/IAIP $30 \mathrm{mg} / \mathrm{kg}$
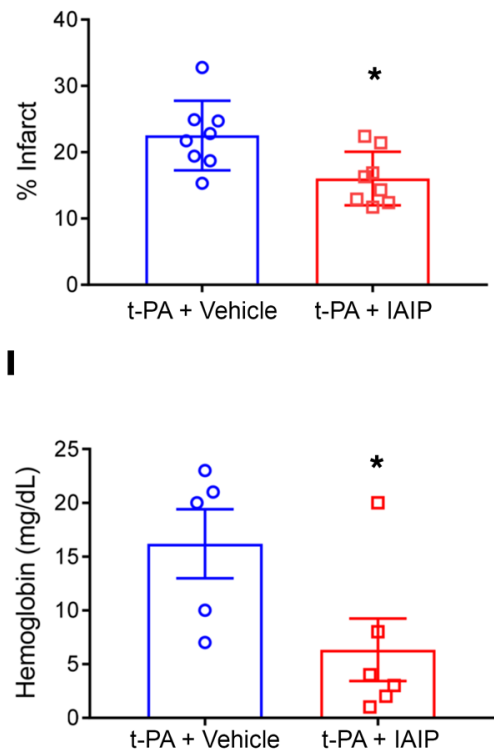

G

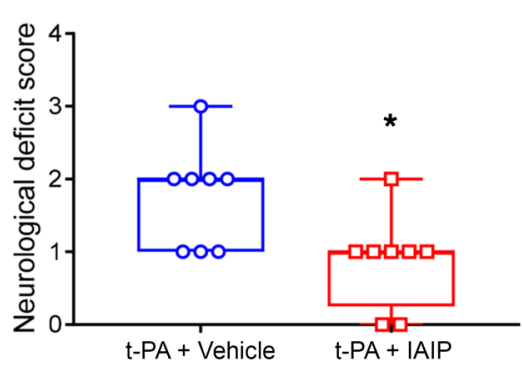

J

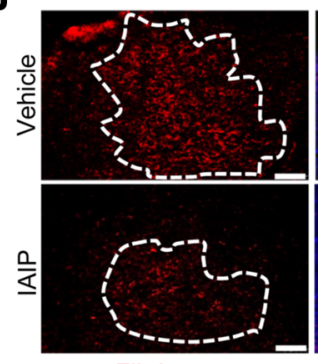

Fibrinogen

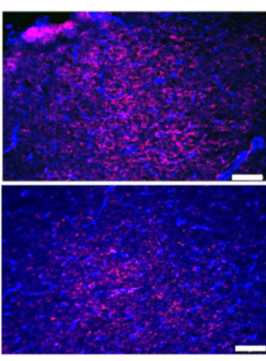

Lectin + Fibrinogen
H

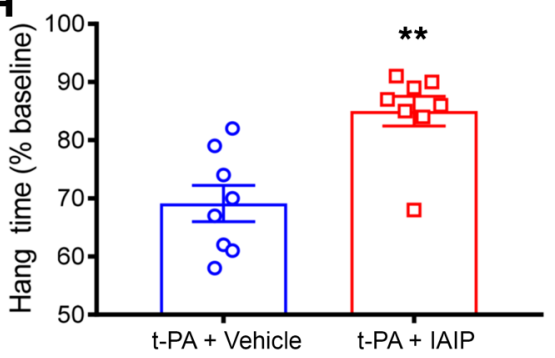

$\mathbf{K}$

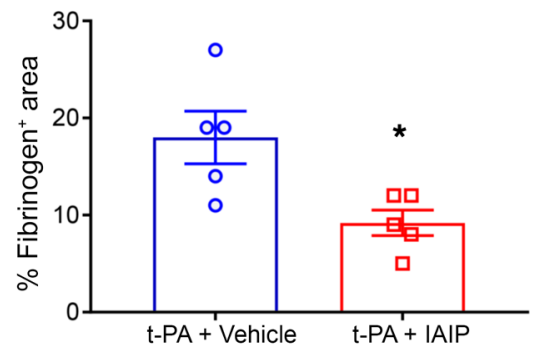

Figure 6. IAIP treatment confers significant protection in both a permanent occlusion model and a thromboembolic stroke model. (A) Young male mice were subjected to permanent MCAO followed by treatment with IAIP or vehicle. (B) Infarct quantification after permanent MCA occlusion showed neuroprotection in the cortex $\left({ }^{*} P=0.0185\right)$, striatum $\left({ }^{*} P=0.0054\right)$, and total hemisphere $\left({ }^{*} P=0.0111\right)$ at 48 hours after stroke with IAIP compared with the vehicle group by 2 -sample $t$ tests adjusted for multiple testing. (C) NDS 24 hours after stroke with IAIP and vehicle treatment $(P=0.0706$, Wilcoxon's rank sum test). (D) IAIP-treated mice had a significantly longer latency to fall in the grip test 48 hours after stroke $\left({ }^{*} P=0.0378, t\right.$ test). (E) Young male mice subjected to embolic stroke followed by t-PA administration were randomly assigned to IAIP or vehicle treatment as shown in the timeline. (F) Mice that received IAIP in combination with t-PA had reduced infarct compared with mice treated with t-PA and vehicle after thromboembolic stroke (mean \pm SEM; ${ }^{*} P=0.0290$, 2-sample $t$ test). (G) Mice treated with t-PA plus IAIP after thromboembolic stroke demonstrated a significantly improved NDS 24 hours after stroke ( ${ }^{*} P=0.046$, Wilcoxon's rank sum test). (H) IAIP-treated mice had a longer latency to fall in the hang-wire test 48 hours after stroke $(* * P=0.0015$, $t$ test). (I) Mice that received IAIP had significantly reduced hemoglobin levels in the ipsilateral hemisphere ( $P=0.0487, t$ test). (J) Representative immunohistochemistry images analyzed 48 hours after thromboembolic stroke showed that IAIP reduced BBB leakage, as seen by fibrinogen immunoreactivity (red), counterstained with lectin (blue). Scale bars: $75 \mu \mathrm{m}$. (K) Quantification of fibrinogen immunoreactivity in the ipsilateral hemisphere of mice treated with t-PA plus IAIP compared with vehicle-treated mice (mean $\pm \mathrm{SEM} ;{ }^{*} P=0.0196, t$ test).

As stroke is a sexually dimorphic disease, following Stroke Therapy Academic Industry Roundtable (STAIR) criteria, we further tested whether IAIP treatment also confers neuroprotection in aged female subjects. Using a design similar to that used for males, aged female mice (18-20 months) were subjected to MCAO and treated with delayed IAIP (30 mg/ $\mathrm{kg}$ or $45 \mathrm{mg} / \mathrm{kg}$ ) or vehicle at 6 hours, 18 hours, day 2, and day 3 after stroke onset. Female mice receiving IAIP (both 30 and $45 \mathrm{mg} / \mathrm{kg}$ ) also had significantly 
reduced total hemispheric infarct volumes compared with vehicle-treated mice $(P=0.0143,30 \mathrm{mg} / \mathrm{kg} ; P=0.0019,45 \mathrm{mg} / \mathrm{kg})$ when analyzed at post-stroke day 7 (Figure $7 \mathrm{H})$. Aged female mice did not show a significant difference in mortality ( 4 mice died in the vehicle group and 3 died in each of the IAIP treatment groups at day $7 ; P=0.9161,30 \mathrm{mg} / \mathrm{kg} ; P=0.7742,45 \mathrm{mg} / \mathrm{kg})$. IAIP-treated females also had significantly improved NDSs at post-stroke day $5(P=0.0058,30 \mathrm{mg} / \mathrm{kg} ; P=0.0039,45 \mathrm{mg} / \mathrm{kg}$; Figure $7 \mathrm{I})$.

IAIP treatment significantly downregulates complement receptor C5aR1 in brain and blood. In order to further explore mechanisms by which IAIPs confer benefit following ischemic stroke, we performed a comprehensive multiplex gene expression analysis examining genes related to neuroinflammation. Genes related to complement activation were upregulated in the brain tissue of mice after stroke compared with sham levels (Figure 8A). The complement pathway was found to be the most differentially expressed gene (DEG) pathway following IAIP treatment compared with vehicle treatment in brain (Figure $8 \mathrm{~B}$ ). NanoString analysis of whole blood revealed that IAIP treatment also reduced the expression of complement pathway genes in the blood in comparison with vehicle-treated mice, with complement component integrin $\alpha_{M}$ (ITGAM) and C5a anaphylatoxin chemotactic receptor 1 (C5aR1) identified as the top DEGs following IAIP treatment (Figure 8C). To further investigate the underlying association between DEGs, a protein interaction network of the top 60 DEGs was constructed using the STRING database (Figure 8D). Mapped results identified C5aR1 as a gene of interest, demonstrating high interactions with several top DEGs (Figure 8D). We further confirmed these changes by independent quantitative PCR (qPCR) analysis (see Supplemental Table 4 for primer sequences), which found that C5aR1 expression was significantly reduced in both brain $(P=0.0281)$ and blood ( $P=0.0249)$ of IAIP-treated mice compared with mice receiving vehicle control (Figure $8 \mathrm{E}$ ). To test whether changes in circulating C5aR1 are simply due to differences in infarct size, in a subcohort of mice we drew blood via cheek bleed before administration of IAIP at 6 hours after stroke and 6 hours after IAIP treatment. Results suggested that C5aR1 was significantly downregulated following IAIP treatment $(P=0.0064$; Supplemental Figure 5). Since C5aR1 is highly expressed in neutrophils and plays an important role in neutrophil recruitment to sites of injury, we found that C5aR1 expression on neutrophils was significantly reduced after IAIP treatment by flow cytometry $(P=$ 0.0072; Figure 8F). Next, we used the C5ar1tm1Cge/J (C5aR1-/-) knockout mouse model to investigate whether $\mathrm{C} 5 \mathrm{aR} 1$ plays a role in the beneficial effects of IAIP in ischemic stroke. As BALB/c mice are sensitive to ischemia (40), we used a 30-minute MCAO model for this experiment to minimize mortality. We found that vehicle-treated $\mathrm{C} 5 \mathrm{aR} 1^{-/-}$mice had significantly smaller infarcts compared with strain-matched vehicle-treated wild-type mice, in cortical $(P=0.0015)$, striatal $(P=0.0023)$, and total hemispheric infarcts $(P=0.0002)$. While wild-type mice treated with IAIP demonstrated significant neuroprotection (Supplemental Figure 6), mice lacking C5aR1 did not show any additional synergistic neuroprotective effects with IAIP treatment, suggesting that IAIP's beneficial effects are mediated at least in part by C5aR1 (Figure 8, G and H).

\section{Discussion}

Our studies confirmed that endogenous circulating IAIP levels are significantly reduced 24 hours after ischemic stroke, both in stroke patients and after experimental stroke in mice. Our findings illustrate that exogenous supplementation of IAIPs can significantly reduce infarct size in the transient MCAO model of stroke, regardless of whether IAIPs were administered at the time of injury or delayed for 6 hours after stroke onset. We confirmed IAIP-mediated protection in a permanent model of ischemic stroke without reperfusion, in a distal stroke model, and in aged females. Next, we found that IAIP and t-PA in combination significantly reduced striatal infarct compared with t-PA alone, and reduced hemorrhagic transformation, maximizing the potential clinical utility of IAIPs in the treatment of human stroke. Finally, by performing multiplex gene expression analysis and by using a C5aR1-knockout mouse model, we determined that this complement receptor plays a role in IAIP neuroprotection.

Previous studies have shown that bikunin is protective against ischemia/reperfusion injury in the intestine $(27,41)$, liver (42), kidney (43), heart (44), and lung (41). Bikunin is the light chain fragment of IAIP and can be isolated from human urine. Bikunin, also known as urinary trypsin inhibitor, has a relatively short half-life (45). IAIPs have been shown to increase survival, in part by effectively downregulating proinflammatory and upregulating antiinflammatory cytokines in sepsis models $(42,46,47)$. Earlier studies reported neuroprotective effects of bikunin in both global and focal stroke $(27,48)$. However, these studies used the short-acting light chain fragment, gave high doses with continuous i.v. infusion, only examined young males, and, most importantly, began dosing the drug before induction of ischemia.

Emerging literature suggests that IAIPs form a complex with histones $(16,49)$, likely via glycosaminoglycan moieties, and neutralize the cytotoxic effects of histones, preventing secondary damage to tissues. These extracellular histones act as damage-associated molecular patterns (DAMPs) and are involved in aging and the pathogenesis of stroke (50). Previous studies have reported complement inhibition as an important underlying mechanism in beneficial effects of IAIPs in lung injury and sepsis models. Our work demonstrates that IAIPs can significantly reduce injury in the ischemic brain of adult and aging animals, even if treatment is delayed for 6 hours after stroke onset. This protection is durable and leads to long-term improvements in motor and cognitive deficits after injury in rodent models, which was associated with a decrease in systemic and brain inflammation.

The early response to ischemic stroke is characterized by necrotic cell death resulting from excitatory neurotoxicity, production of free oxygen radicals, oxidative and nitrosative stress, and mitochondrial dysfunction. As this "core" injury is rapid and irreversible, selective targeting of these mechanisms has not yielded effective neuroprotective strategies in clinical trials (51). However, delayed secondary apoptotic cell death occurs in the penumbra, leading to the release of DAMPs and activation of neuroinflammation. Unfortunately, the vast majority of preclinical neuroprotective agents to date have been developed and tested in young, healthy animals owing to the significant cost and higher mortality seen in aged animals. Translation of these potential therapies into clinical populations has been unsuccessful partially because of lack of validation in aged animal models. 
A

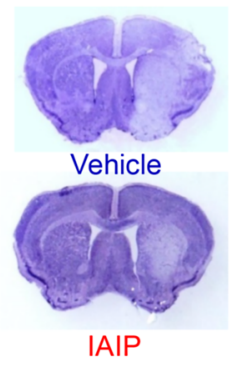

B

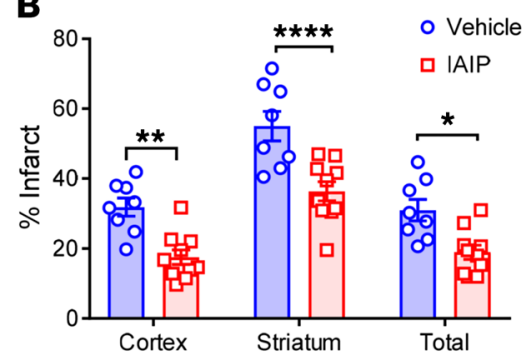

C

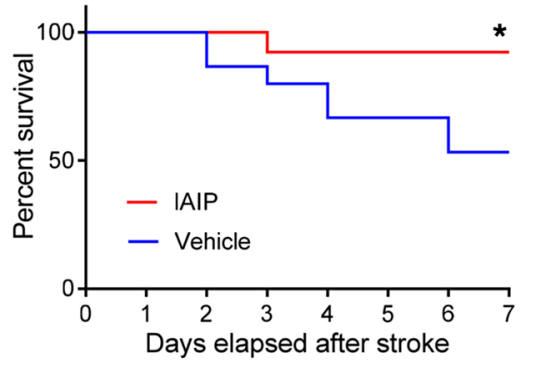

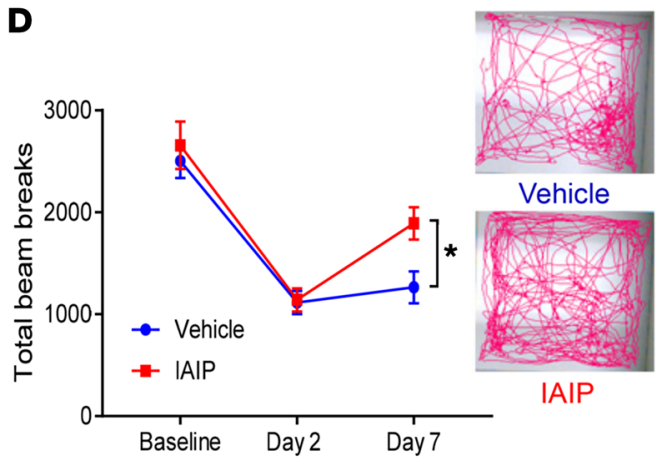

E

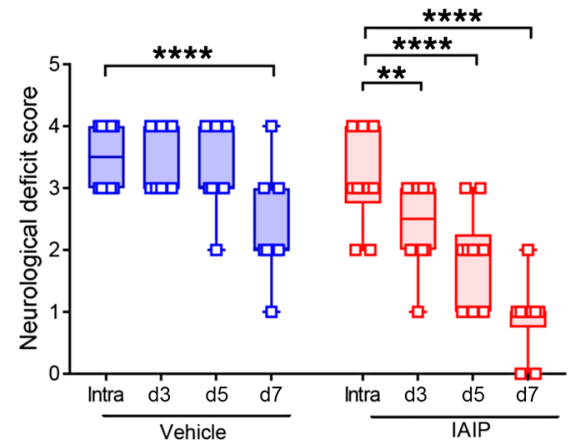

$\mathbf{F}$

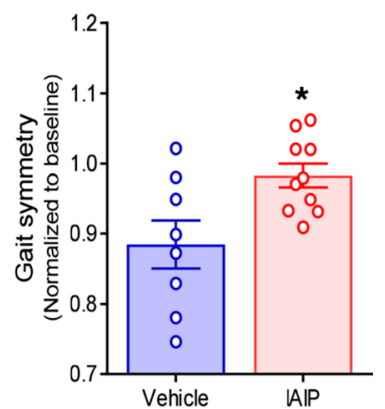

G

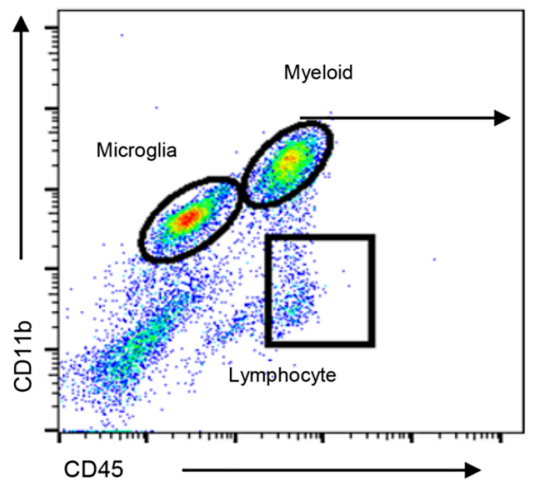

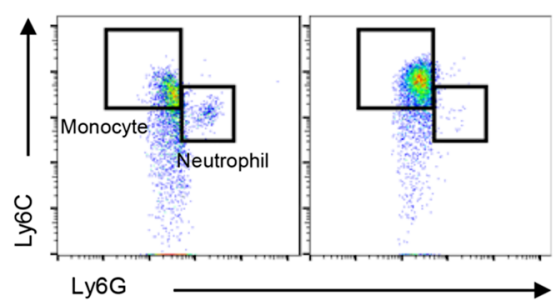

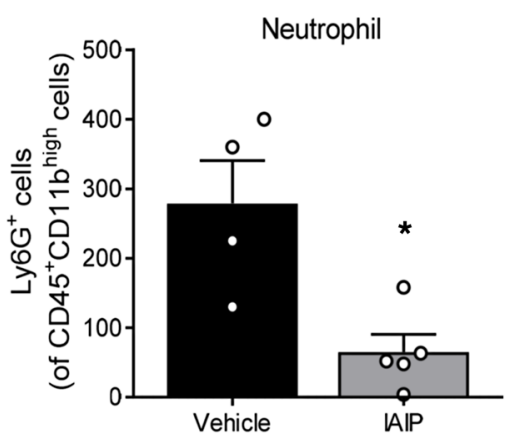

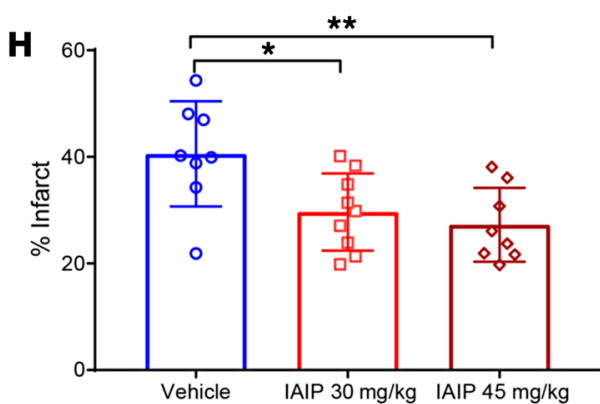

I

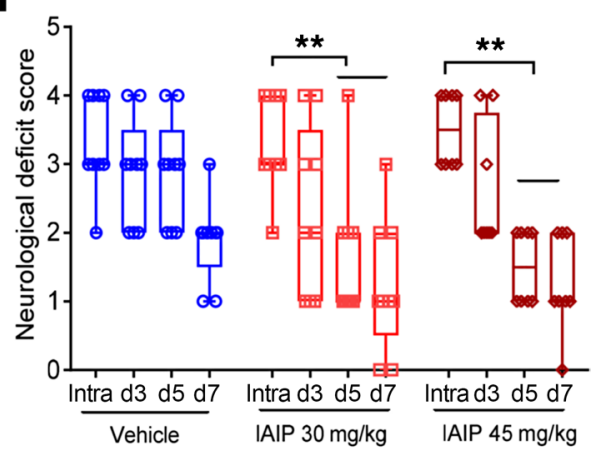

Figure 7. Delayed IAIP treatment reduces infarct size, decreases mortality, and improves functional outcome in aged mice after stroke. (A) Representative images showing protective effects of IAIP even with delayed treatment in aged male mice analyzed at post-stroke day 7. (B) Infarct quantification demonstrating reduced infarct size with IAIP treatment compared with vehicle control: cortex $\left({ }^{* *} P=0.0020\right)$, striatum $\left({ }^{* * *} P<\right.$ $0.0001)$, and total hemisphere $\left({ }^{*} P=0.0113\right.$ ) (2-sample $t$ test adjusted for multiple testing). (C) IAIP treatment significantly improved survival rates $\left({ }^{*} P=0.0337\right.$, log-rank test). (D) Exogenous IAIP treatment significantly improved locomotor recovery after stroke $\left({ }^{*} P=0.0323\right.$, repeated-measures 2-way ANOVA adjusted for multiple testing). (E) Significant improvement in NDS in mice treated with IAIP was observed at day $3\left({ }^{* *} P=0.0013\right.$ ), day $5\left({ }^{* * *} P<0.0001\right)$, and day $\left.7{ }^{* * * *} P<0.0001\right)$, while vehicle-treated mice expressed significant recovery only at day $7\left({ }^{* * * *} P<0.0001\right)$, by mixed-effects model. (F) Aged mice receiving delayed IAIP treatment demonstrated restored gait symmetry compared with mice that received vehicle by post-stroke day 7 ( ${ }^{*} P=0.0147, t$ test). (G) IAIP significantly reduced stroke-induced neutrophil infiltration into the ipsilateral hemisphere measured at 3 days after stroke, shown as representative flow plots and quantification data $\left({ }^{*} P=0.0105, t\right.$ test). (H) Infarct analysis in aged female mice after 60-minute MCAO showed a reduction in the total hemispheric infarct with $30 \mathrm{mg} / \mathrm{kg}\left({ }^{*} P=0.0143\right)$ and $45 \mathrm{mg} / \mathrm{kg}\left({ }^{* *} P=0.0019\right)$ at 7 days after stroke compared with the vehicle group by 1-way ANOVA adjusted for multiple testing. (I) NDS also showed significant early improvement with $45 \mathrm{mg} / \mathrm{kg}$ IAIP treatment as early as day 5 after stroke, while vehicle treatment was not statistically significant $(* * P=0.0058$ with $30 \mathrm{mg} / \mathrm{kg}$, ${ }^{* *} P=0.0039$ with $45 \mathrm{mg} / \mathrm{kg}$, mixed-effects model). 
Brain injury results in a robust systemic inflammatory response, leading to production of proinflammatory cytokines and activation and trafficking of immune cells from the bone marrow to the brain $(7,8,52)$. The subsequent elevation of proinflammatory cytokines and excess complement activation lead to recruitment of peripheral inflammatory cells, specifically neutrophils, to the injured site (53). These infiltrated neutrophils are capable of inducing a second wave of inflammatory response by promoting recruitment of inflammatory monocytes (54). In addition, recent studies have shown that IAIPs have important effects on human neutrophils in vitro, preventing neutrophil activation, maintaining neutrophil shape, and inhibiting adhesion to vascular endothelial cells by suppressing ROS release (21). Many of these beneficial effects of IAIPs on systemic neutrophils observed in vitro could also contribute to the reduced stroke injury. Furthermore, the release of cytotoxic substances such as extracellular histones from neutrophils and other DAMPs further increases the inflammatory response and impairs recovery. Although the smaller infarcts in mice treated with IAIPs may also be a factor contributing to this faster recovery, previous studies suggest that infarct size does not necessarily correlate to behavioral functional deficits (55), and the deficits even at 48 hours after stroke were similar in both vehicle- and IAIP-treated mice in the corner test. Importantly, the behavioral benefit of IAIP treatment continued to be significant on day 7 after stroke and remained significantly better even at 28 days after stroke in young male mice, suggesting that this treatment targets the secondary inflammatory phase that continues after stroke. Thus, IAIP treatment provides long-term benefits after stroke. Several behavioral tests were used to evaluate functional and behavioral recovery based on the sensitivity of the test in the different stroke models. Mice treated with IAIPs had significantly improved cognitive outcomes as assessed by the novel object recognition test and the Barnes maze compared with vehicle-treated mice. In addition to improvement of gross neurological deficit scores, aged mice treated with IAIPs demonstrated more rapid recovery in locomotor activity in the open field test by day 7. IAIP-treated mice also had significantly improved somatosensory function in the adhesive-tape removal test at day 7 even after a mild distal stroke, demonstrating that the beneficial effect of exogenous IAIPs in aged mice was not due to a survival effect. These observations further strengthen the possibility that IAIP treatment could enhance post-stroke recovery.

We then investigated whether IAIPs can confer neuroprotection in the absence of reperfusion and in combination with t-PA, as the use of multiple stroke models helps better mimic the heterogeneous clinical stroke population. Indeed, based on our results from the permanent occlusion model (MCAO remained occluded for 48 hours without reperfusion), we concluded that the cascade of protective mechanisms initiated by exogenous IAIP treatment led to beneficial effects even in the absence of reperfusion. The only currently available pharmacological treatment for stroke, $\mathrm{t}-\mathrm{PA}$, is a serine protease, and currently there is no information regarding how IAIP treatment could alter its function after stroke. Our studies found significant neuroprotective effects with delayed IAIP treatment even in combination with t-PA, and animals treated with IAIP had significantly reduced hemoglobin in the ipsilateral hemisphere, suggesting that IAIPs may also reduce risk of hemorrhagic transformation after treatment with t-PA. Delayed IAIP administration did not significantly change coagulation time in comparison with vehicle groups after t-PA administration in a tail bleed assay, suggesting that the beneficial effects are not directly mediated by enhanced anticoagulant activity. The beneficial effects of IAIPs were found even when they were administered 6 hours after stroke onset, 90 minutes after the longest t-PA window has closed ( 4.5 hours) in clinical practice. This is especially important, as it suggests that IAIPs could confer additional beneficial effects even in patients who receive t-PA, potentially via other mechanisms beyond the regulation of serine protease activity.

Next, we performed gene analysis using NanoString on both blood and brain samples collected at 72 hours after onset of stroke from aged animals treated with vehicle or IAIP starting at 6 hours after stroke. We used this panel to identify and exploit genes that are activated with stroke and normalized with IAIP treatment. Consistent with previous studies in young animals $(53,56,57)$, we found that the complement pathway was the most significantly altered pathway after stroke in aged animals. Further analysis and independent qPCR validation revealed that the complement receptor C5aR1 was the target most consistently suppressed by IAIP treatment in both the blood and the brain in aged animals. To confirm that the low C5aR1 expression was not due to the smaller injury in IAIP-treated animals, using a separate cohort of mice, we tested blood C5aR1 levels from the blood collected from cheek bleeds prior to administration of IAIP and following IAIP administration.

The complement system is a vital arm of the innate immune system but has functions that extend far beyond the realm of infectious disease. Recent studies suggest that enhanced complement activation can adversely affect host cells and induce or exacerbate immune and inflammatory responses, contributing to microglial activation, neutrophil recruitment, and enhanced cell death (53, $56,57)$. Systemic activation of complement leads to activation and recruitment of myeloid cells via C5a/C5aR1 signaling in inflammatory lung diseases, including COVID-19 (58). Our data demonstrate an increase in both circulating and central C5aR1 levels after stroke. Moreover, C5a/C5aR1 signaling acts as a chemoattractant for neutrophils and monocytes to the sites of injury or inflammation and is involved in autoimmunity, neutrophil dysfunction, and increased production of IFN- $\gamma$ and ROS in neutrophils $(59,60)$. Previous in vitro studies using cultured neurons have reported increased C5a expression with oxygen glucose deprivation (OGD) and stress (53), while C5aR1 deletion prevented OGD-induced neuronal apoptosis. Inhibition of C5aR1 has been shown to attenuate apoptotic neuronal cell death in vitro (61), reduces infarct volume in in vivo stroke models $(62,63)$, and improves recovery in a spinal cord injury model (64). Here, we tested whether IAIP treatment alters C5aR1 expression on neutrophils and whether IAIP-induced neuroprotection is mediated via C5aR1 using C5aR1-knockout mice. IAIP treatment inhibited C5aR1 expression in both the brain and the blood after stroke.

Furthermore, deletion of C5aR1 was neuroprotective after stroke; the total lesion volume was lower in C5aR1-knockout mice compared with strain-matched wild-type mice. However, IAIP administration led to no additive benefit in C5aR1-knockout mice. A floor effect is possible, but the hemispheric infarct in these mice is larger than the infarct seen in the distal model, where IAIPs 
A

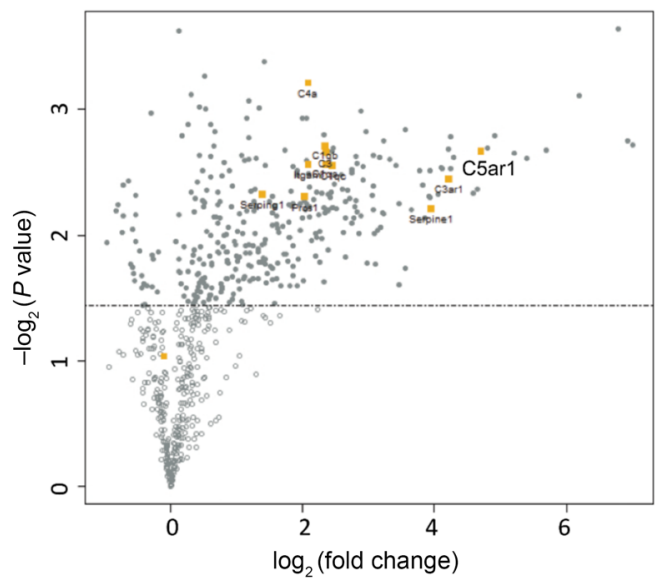

B

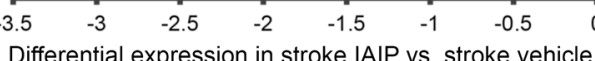

D

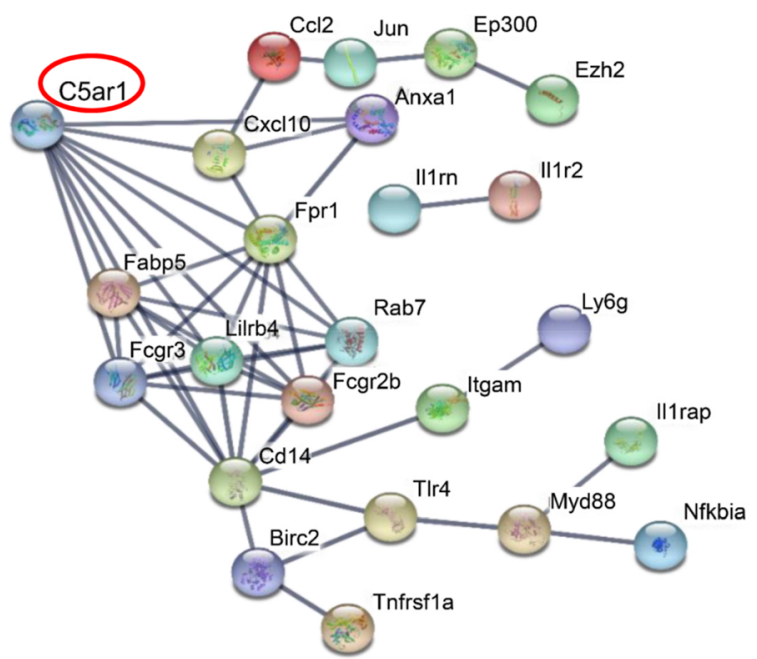

\section{E}

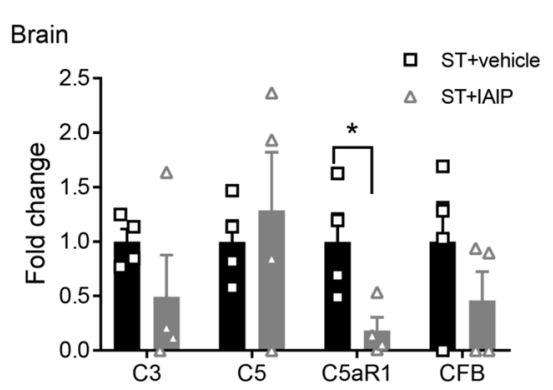

Btose

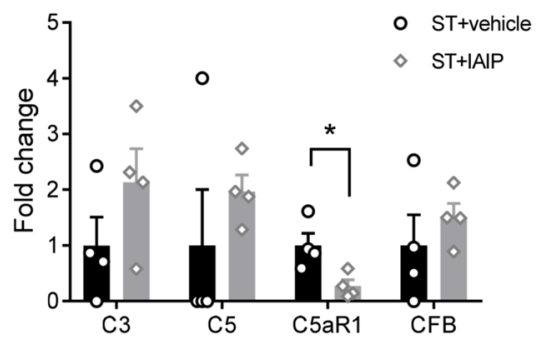

$\mathbf{F}$

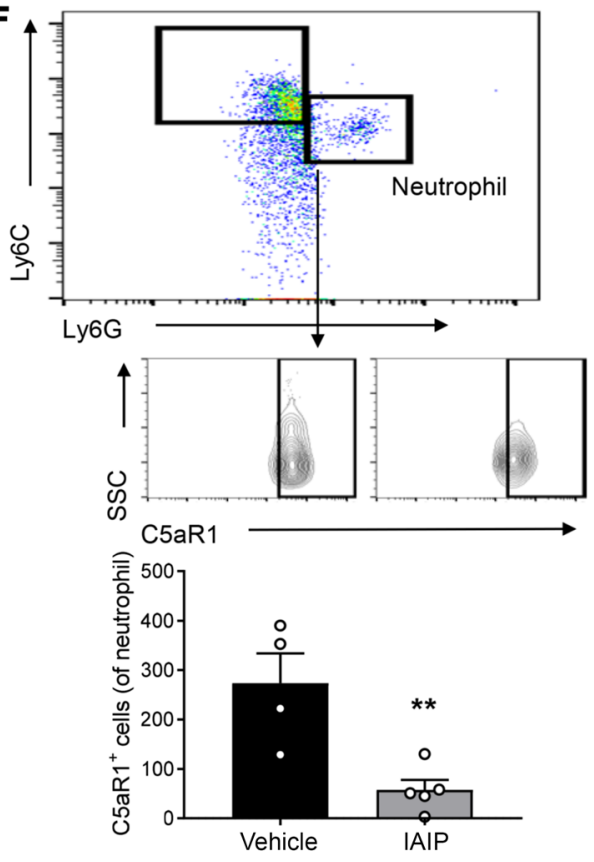

G
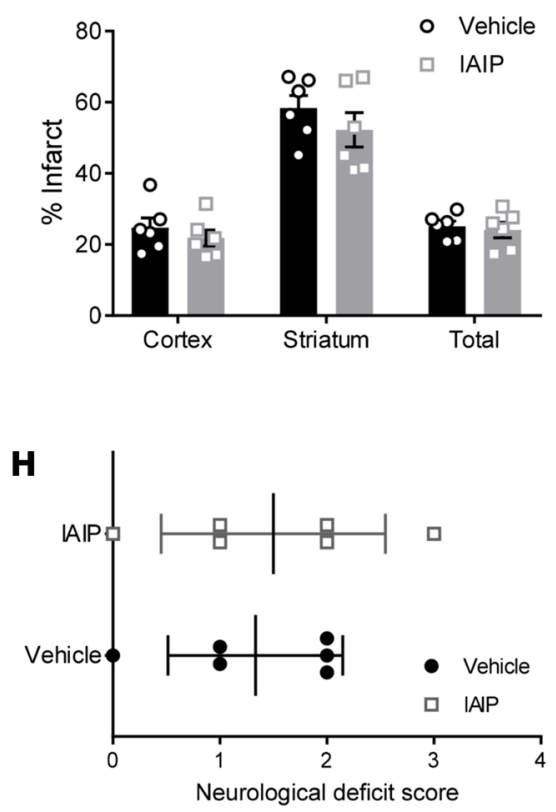
Figure 8. IAIP mediates its beneficial effects by suppressing C5aR1 expression in both blood and brain. (A-C) NanoString analysis. (A) Analysis of stroke and sham brains revealed that complement activation is highly upregulated after stroke ( $n=3$ per group). (B) Vehicle- versus IAIP-treated groups revealed that the most predominant DEGs are mapped to complement suppression in the brains of aged stroke animals, analyzed at 72 hours after stroke ( $n=3$ per group). (C) IAIP also reduced stroke-induced complement pathway in blood of aged stroke mice ( $n=3$ per group). Complement changes are presented as heatmap. (D) STRING analysis of the top 60 DEGs from brain is plotted. C5aR1 showed a strong association with several of these mRNAs. (E) Independent analysis and validation of complement changes revealed that $\mathrm{C5} \mathrm{aR} 1$ is significantly reduced in both brain ( $\left.{ }^{*} P=0.0281\right)$ and blood $\left({ }^{*} P=0.0249\right)$ of IAIP- versus vehicle-treated groups (2-sample $t$ test). (F) IAIP treatment significantly reduced C5aR1 expression on infiltrating neutrophils in aged male brains $\left({ }^{* *} P=0.0072, t\right.$ test). (G and $\mathbf{H})$ C5aR1-knockout mice had no significant benefit from IAIP treatment in terms of cortex $(P=0.4433)$, striatum $(P=0.3325)$, and total hemisphere $(P=0.6962)$ infarcts ( 2 -sample $t$ tests adjusted for multiple testing) (G) and NDS (accessed by Wilcoxon's rank sum test) (H), suggesting that IAIP's beneficial effects are mediated by C5aR1 signaling. Data are presented as mean \pm SEM.

were protective. In addition, there was no reduction in striatal infarction, which was quite large, making a floor effect less likely. Our findings of significantly reduced infarct size in C5aR1-knockout mice after stroke, and the lack of beneficial effect of IAIP treatment, suggest that IAIP mediates its beneficial effects at least in part by suppressing C5aR1 expression and thus reducing neutrophil infiltration. The mechanistic relationship between IAIPs and C5aR1 will require further investigation.

Identification of clinical biomarkers for stroke outcomes and effective pharmacological treatments to enhance stroke recovery are urgently needed. Our clinical data strongly suggest that IAIP levels are significantly lower in stroke survivors at 24 hours compared with clinical controls. Consistently, we found that stroke survivors with higher circulating IAIP levels had significantly lower levels of circulating TNF- $\alpha$, IFN- $\gamma$, and CCL11, and mice that received IAIP had higher antiinflammatory and lower proinflammatory cytokines, indicating that IAIPs may play an important role in reducing inflammation. Reduced levels of these inflammatory biomarkers in stroke patients are associated with better survival rates after stroke (65). This also suggests that monitoring stroke-induced loss of IAIPs may allow us to develop a personalized dosing regimen for individual patients by following their levels of IAIPs or IAIP-histone complexes and supplementing exogenous IAIPs when levels decline.

In conclusion, our findings suggest that IAIP levels decline after stroke in mice and humans, and that exogenous administration of IAIPs represents a powerful potential therapeutic strategy in the treatment of ischemic stroke. This study demonstrates that both early and delayed exogenous IAIP administration following stroke can significantly reduce injury-related tissue loss and improve post-stroke recovery in the MCAO ischemia/reperfusion model of stroke. Furthermore, our results indicate significant beneficial effects of IAIPs in permanent ischemia, where there is no reperfusion, and when the occluded blood vessel is recanalized by t-PA, two common scenarios seen in clinical practice. In addition, correlative analysis from human samples suggests that higher plasma IAIP levels are associated with reduced inflamma- tion, indicating that IAIPs could potentially represent a predictive biomarker and an effective therapeutic treatment for ischemic stroke. Our studies using aged animal models, human clinical samples, and therapeutic testing of IAIPs in several clinically relevant stroke models further strengthen the clinical translatability of these findings.

\section{Methods}

Further information can be found in Supplemental Methods.

Human plasma-derived IAIPs. IAIPs were extracted from fresh-frozen human plasma (Rhode Island Blood Center) as previously described $(66,67)$. The purification process consisted of a scalable capture step of IAIPs using either monolithic strong anion-exchange chromatographic support CIMmultus QA (BIA Separations, Ajdovščina, Slovenia) or TOYOPEARL GigaCap Q-650M (Tosoh Bioscience) and then a polishing step using a proprietary synthetic chemical ligand affinity chromatographic support (Astrea Bioseparations). The bound proteins were eluted from the columns by buffers containing high salt (up to $750 \mathrm{mM} \mathrm{NaCl}$ ). The process resulted in a high-yield, high-purity $(>90 \%)$, and biologically active IAIP. Eluted proteins were concentrated and buffer-exchanged using a tangential flow filtration device (Labscale, MilliporeSigma). Analyses of the purity and biological activity of the IAIP were performed using SDS-PAGE, Western immunoblot, protein assay, and ELISA. The biological activity was measured based on the ability of IAIP to inhibit the hydrolysis of the substrate $\mathrm{N}$-benzoyl-L-arginine- $p$-nitroaniline $\mathrm{HCl}$ (L-BAPNA, MilliporeSigma) by trypsin. The highly purified IAIPs were frozen until use and reconstituted in sterile PBS before administration to experimental animals.

Human plasma samples. Ischemic stroke was defined as an acute neurological deficit with CT- or MRI-confirmed cerebral infarction. Exclusion criteria for the study included ischemic stroke secondary to iatrogenic causes; stroke due to dissection, underlying malignancy, or autoimmune disorders; and equivocal imaging. Blood was drawn at $24 \pm 6$ hours after stroke, followed by centrifugation and removal of the plasma fraction for storage at $-80^{\circ} \mathrm{C}$ for future analysis $(n=51)$. Plasma obtained from non-stroke subjects served as control $(n=39)$. Human-subject data for the plasma samples are included in Supplemental Table 2.

Human brain sections. Peri-infarct tissue sections from 13 patients with ischemic stroke in the middle cerebral artery territory were selected (age $80.8 \pm 2.6$, mean \pm SD) and compared with sections from male age-matched controls without evidence of stroke (age 75.4 \pm 3.8). All slides were analyzed by an investigator blinded to case demographics and stroke condition (Supplemental Table 3).

Immunofluorescence staining. Human brain sections were deparaffinized in xylene and rehydrated through a graded series of ethanol baths. After antigen retrieval with sodium citrate buffer (68), nonspecific antibody binding was blocked by incubation of the sections with $3 \%$ BSA in PBS for 60 minutes. After incubating with primary antibodies (1:100; polyclonal anti-IAIP, R22C) in TBS plus $0.25 \%$ Triton $\mathrm{X}-100$ overnight at $4^{\circ} \mathrm{C}$, the sections were washed with PBS for $5 \mathrm{~min}$ utes 3 times. Subsequently, anti-rabbit 488 (1:1000; catalog 406416, BioLegend) was used as secondary antibody for 60 minutes at room temperature and washed with PBS for 10 minutes 3 times. Images were collected using a Leica confocal microscope. All slides were analyzed using Image (Colocalization Colormap; NIH) by an investigator blinded to case demographics and stroke condition. 
Mice. Wild-type C57BL/6 young male (8-12 weeks) and C57BL/6 aged male and female (18-20 months) mice were purchased from Charles River Laboratories. Young (8-12 weeks) C5ar1tm1Cge/J $\left(\mathrm{C} 5 \mathrm{aR} 1^{-/-}\right)$male mice and control mice were purchased from The Jackson Laboratory. All mice used for these experiments were housed 5 per cage in standard facilities with a 12-hour light/12-hour dark schedule in a temperature- and humidity-controlled vivarium and ad libitum access to food and water. Surgical procedures were performed following the criteria derived from the Stroke Therapy Academic Industry Roundtable (STAIR) group guidelines for preclinical evaluation of stroke therapeutics, as described previously (31). Animals were randomly assigned to treatment groups. A total of 14 mice were excluded from the study analysis, because of either subarachnoid hemorrhage $(n=11)$ or excessive loss of body weight after stroke $(n=3)$. Mice that died during experiments are reported in mortality curves but excluded from behavioral and histological analysis. Infarct analysis and behavioral testing were performed by an investigator blinded to treatment conditions.

Middle cerebral artery occlusion models. Focal transient cerebral ischemia was induced in mice by right middle cerebral artery occlusion (MCAO) followed by reperfusion as described previously $(31,69)$. Reperfusion was not performed in the permanent occlusion cohort. Extreme care was taken to minimize pain and discomfort to the animals. Anesthesia was induced with $4 \%$ isoflurane, and adequate sedation was confirmed by tail pinch for all surgical procedures. Surgery was performed under $1 \%-2 \%$ continuous isoflurane. At the end of ischemia (30/60/90 minutes of MCAO depending on the cohort), the animal was briefly reanesthetized, and reperfusion was initiated by filament withdrawal. Cortical perfusion (laser speckle/Doppler flowmetry [LDF]) was evaluated throughout MCAO and early reperfusion, and body temperature was maintained at approximately $37^{\circ} \mathrm{C}$ as described previously (69). Mice were randomly assigned to receive either IAIP or vehicle (PBS) treatment by i.p. injection, and were followed for up to 30 days for behavioral testing prior to euthanasia.

Flow cytometry. Blood was collected by cardiac puncture with heparinized needles from deeply anesthetized mice. Mice were then transcardially perfused with $60 \mathrm{~mL}$ cold, sterile PBS before the removal of the brain and preparation of the ipsilateral stroke hemisphere for analysis by flow cytometry. A gating strategy was applied as previously described $(39,70)$. Cell type-matched fluorescence minus one controls were used to determine the positive gating for each antibody. Isolated cells were stained with the following antibodies: anti-CD45 (clone 30-F11, Invitrogen, 48-0451-82), anti-CD11b (clone M1/70, Invitrogen, 53-0112-82), anti-Ly6C (clone HK1.4, Invitrogen, 47-5932-80), anti-Ly6G (clone 1A8, BioLegend 127616), anti-C5aR1 (clone 10/92, Invitrogen, MA1-81761); and an amine-reactive Live/Dead Aqua viability stain (Invitrogen, L34966) was used to identify live cells. Neutrophils were identified as $C D 45^{+} C D 11 b^{\text {hi- }}$ Ly $6 \mathrm{C}^{\mathrm{lo}} \mathrm{Ly} 6 \mathrm{G}^{+}$. Cell counts obtained from the ischemic hemispheres of vehicle- and IAIP-treated animals are representative of 2 biological replicates. Flow cytometry was performed on a CytoFLEX (Beckman Coulter) flow cytometer, and data were analyzed using FlowJo software (Tree Star Inc.).

NanoString analysis and real-time qPCR. Blood and brains were harvested from aged male mice 72 hours after ischemia/reperfusion following MCAO. Total RNA from brain tissues was isolated using Trizol (Invitrogen), and whole-blood RNA was isolated according to the manufacturer's protocol using a mouse RiboPure-Blood RNA Isolation kit (Invitrogen). cDNA was synthesized using 500 ng of RNA with iScript Reverse Transcription Supermix for RT-qPCR kit (Bio-Rad).

Gene expression analysis was performed using RNA extracted from blood and brain tissue and the NanoString nCounter Neuroinflammation Panel (NanoString Technologies Inc.), per the manufacturer's instructions. Samples were processed on the nCounter MAX Analysis System, following quality control validations. Raw data were collected and processed using nSolver Analysis Software version 4.0 with the recommended threshold settings for background corrections and statistical analyses. Heatmaps were generated using nSolver software. Gene interaction analysis was performed using the online Search Tool for the Retrieval of Interacting Genes/Proteins (STRING, version 11.0; https://string-db.org/) with the minimum required interaction score set at 0.900 (highest confidence). For independent qPCR, $\beta$-actin was used to normalize the expression level of mRNAs. qPCR was performed using CFX96 Touch Real-Time PCR Detection System (Bio-Rad) with SsoAdvanced Universal SYBR Green Supermix (Bio-Rad). The thermal cycling protocol used for qPCR was as previously detailed (70).

TTC quantification of infarct volume. Brains were sectioned into five 2-mm coronal sections and incubated with 1\% 2,3,5-triphenyl-tetrazolium chloride (TTC) in saline for 8 minutes at $37^{\circ} \mathrm{C}$ before image acquisition and volumetric quantification as previously described (69).

Terminal histopathology and atrophy quantification. Mice were perfused with PBS containing $1 \%$ heparin followed by $4 \%$ paraformaldehyde. Brains were postfixed in $4 \%$ paraformaldehyde for 6 hours and then placed in sucrose. Sections were then thin-cut at $30 \mu \mathrm{m}$ thickness and collected into 96-well plates for IHC and infarct analysis as previously described (71). To assess changes in hippocampal neuronal density, 4 cresyl violet-stained coronal brain sections per animal were analyzed (bregma coordinates approximately $-1.58 \mathrm{~mm},-2.06 \mathrm{~mm},-2.46$ $\mathrm{mm},-2.92 \mathrm{~mm}$ ) ( $n=5$ per group), and normal and atrophic neurons in the CA1 region of the hippocampus were counted by a blinded investigator. Data are presented as percentage of normal-appearing neurons.

ELISA. Plasma samples from both humans and mice were used to measure the levels of circulating IAIPs. Human plasma samples were collected as described above. Blood from mice was collected with a heparinized syringe at sacrifice via cardiac puncture. Blood was centrifuged at $10,000 \mathrm{~g}$ for 10 minutes at $4^{\circ} \mathrm{C}$, and the plasma layer was collected into separate tubes and stored at $-80^{\circ} \mathrm{C}$ until further analysis. Two separate ELISA kits were used to detect changes in human and murine IAIPs. The competitive human IAIP ELISA used a monoclonal antibody specific against human IAIP (MAb 69.26) and was developed to quantitatively measure IAIP in biological fluids (ProThera Biologics Inc). MAb 69.26 binds only to human IAIP (both IaI and PaI), as the epitope defined by the antibody is located in the light chain subunit and shared by IaI and PaI. However, the monoclonal antibody does not cross-react with murine IAIP. To measure endogenous mouse IAIP levels, a rabbit polyclonal antibody against rodent IAIP was used (PAb-R22, ProThera Biologics Inc.) in a competitive mouse IAIP ELISA. The polyclonal antibody was generated by immunization of rabbits with highly purified rat IAIP. In the competitive IAIP ELISA, biotinylated IAIP was used to compete with IAIP present in plasma samples, whereas in the sandwich IAIP-histone ELISA, a monoclonal anti-histone antibody (against H1 and core proteins; catalog MABE71, 
clone F152.C25.WJJ, MilliporeSigma) that was previously conjugated with biotin was used to detect histone in the captured IAIP-histone complex. The binding of biotinylated molecules was visualized by streptavidin-conjugated HRP, and the color change following TMB substrate was measured spectrometrically at $450 \mathrm{~nm}$. Hemoglobin measurements were obtained from homogenized perfused brains according to the manufacturer's instructions for the Hemoglobin Colorimetric Assay kit (Cayman Chemical Co.) as detailed previously (39).

Open field test. The automated open field test was performed in chambers with infrared beam LEDs to measure locomotor activity as described previously $(68,72)$. Changes in activity were automatically registered via computer, and data were analyzed by an investigator blinded to treatment conditions.

Neurological deficit score. Neurological deficit scoring was performed at day 0 (60 minutes after occlusion) and at day 1 and for several days after surgery according to the following graded scoring system: 0 , no deficit; 1 , forelimb weakness and torso turning to the ipsilateral side when held by tail; 2 , circling to affected side; 3 , unable to bear weight on affected side; and 4, no spontaneous locomotor activity or barrel rolling, as described previously $(68,73)$.

Corner test. The corner test detects integrated sensorimotor function as it involves both stimulation of the vibrissae (sensory/neglect) and rearing (motor response), and was carried out as previously described (72). Twenty trials were performed for each mouse, and the percentage of right turns was calculated.

Statistics. Descriptive statistics (i.e., mean, SEM, median, interquartile range) are provided for variables of interest. Bar charts, box plots, survival plots, or longitudinal mean plots are provided when appropriate (Figures 1-8). For 2-group comparisons, 2-sample, 2-sided $t$ test or Wilcoxon's rank sum test was used depending on the normality of the data distribution. When normality held, 2-sample $t$ test was used; otherwise, Wilcoxon's rank sum test was used. One-way ANOVA or Kruskal-Wallis test was used when there were more than 2 groups. Two-way ANOVA was used for cases with 2 factors such as stroke/sham and IAIP/vehicle. Repeated-measures 2-way ANOVA was used for outcomes measured repeatedly. When there were missing observations, linear mixed models were used. Factors of stroke/sham, IAIP/vehicle, and days are included in the model. All post hoc tests for subgroup comparison or for drug effect on different days were adjusted for multiple testing by Tukey's, Dunnett's, or Holm-Šidák procedures depending on the cases. Log-rank test was used to compare the survival functions between the IAIP and vehicle groups. Linear regression analysis was used to evaluate the correlation of the levels of inflammatory markers and plasma IAIP levels. The ROUT (robust regression followed by outlier identification) method was used to detect outliers (74), and Cohen's $d$ was used to calculate effect size. Animal numbers were determined by power analysis based on a comparison of primary outcomes between stroke and control mice. Experiments and data analyses were performed blinded to surgical conditions and treatments. All analyses were performed in SAS 9.4 (SAS Institute).
Study approval. Human plasma samples were collected under a protocol approved by Hartford Hospital (Hartford, Connecticut, USA; IRB approval no. HHC-2014-0159) in an 868-bed community-based teaching hospital with Joint Commission certification as a comprehensive stroke center. Formalin-fixed, paraffin-embedded human brain tissue sections were obtained from the University of Pittsburgh neurodegenerative brain bank with appropriate ethics committee approval (Committee for Oversight of Research and Clinical Training Involving Decedents). Written informed consent for research and autopsy was obtained from next of kin for all subjects in the study.

All animal work was approved by the Center for Laboratory Animal Medicine and Care at the University of Texas Health Science Center at Houston and the Center for Laboratory Animal Care at the University of Connecticut (Farmington, Connecticut, USA) and was performed in accordance with Institutional Animal Care and Use Committee and NIH guidelines.

\section{Author contributions}

VRV, MR, and LDM designed the study, performed experiments, researched data, and wrote the manuscript. AP, YJL, YX, DCK, JL, AH, and AC performed experiments, helped with analysis, and researched data. JK and LHS helped perform experiments, supplied human samples, and edited the manuscript. LZ performed statistical analysis and helped write the manuscript. JK and BSS helped edit the manuscript. YPL extracted and purified plasma proteins, developed antibodies, performed experiments, and helped write the manuscript.

\section{Acknowledgments}

The authors thank Joseph Qiu and Andre Santoso of ProThera Biologics for their excellent technical assistance in analyzing IAIP and IAIP-histone complex in human and mouse samples (ELISA).

This work was supported by grants from the NIH National Institute of Neurological Disorders and Stroke (NINDS) to LDM (R21-NS087183, R01-NS094543, R37-NS096493), and the American Heart Association (Scientist Development Grant no. 15SDG23250025, to VRV). MR was supported by an F30 grant (F30NS098628) from the NIH-NINDS. This project was supported by the Genomic and RNA Profiling Core at Baylor College of Medicine (to DCK). The University of Pittsburgh brain bank and JK are supported by NIH grants (National Institute on Aging P50-AG005133 and P30-AG066468) and the University of Pittsburgh Brain Institute.

Address correspondence to: Venugopal Reddy Venna, Department of Neurology, McGovern Medical School, University of Texas Health Science Center at Houston, Houston, Texas 77030, USA. Phone: 1.713.500.7037; Email: Venugopal.R.Venna@uth.tmc.edu. YJL's present address is: Solomont School of Nursing, Zuckerberg College of Health Sciences, University of Massachusetts Lowell, Lowell, Massachusetts, USA.
1. Bushnell C, et al. Guidelines for the prevention of stroke in women: a statement for healthcare professionals from the American Heart Association/American Stroke Association. Stroke. 2014;45(5):1545-1588.
2. Virani SS, et al. Heart disease and stroke statistics-2021 update: a report from the American Heart Association. Circulation. 2021;143(8):e254-e743.

3. Musuka TD, et al. Diagnosis and management of acute ischemic stroke: speed is critical. CMAJ. 2015;187(12):887-893.

4. Aleu A, et al. Hemorrhagic complications after off-label thrombolysis for ischemic stroke. Stroke. 2007;38(2):417-422. 
5. Barber PA, et al. Why are stroke patients excluded from TPA therapy? An analysis of patient eligibility. Neurology. 2001;56(8):1015-1020.

6. Romano JG, et al. Outcomes in mild acute ischemic stroke treated with intravenous thrombolysis: a retrospective analysis of the Get With the Guidelines-Stroke registry. JAMA Neurol. 2015;72(4):423-431.

7. Anrather J, Iadecola C. Inflammation and stroke: an overview. Neurotherapeutics. 2016;13(4):661-670.

8. Dziedzic T. Systemic inflammation as a therapeutic target in acute ischemic stroke. Expert Rev Neurother. 2015;15(5):523-531.

9. Xu J, et al. Extracellular histones are mediators of death through TLR2 and TLR4 in mouse fatal liver injury. J Immunol. 2011;187(5):2626-2631.

10. Xu J, et al. Extracellular histones are major mediators of death in sepsis. Nat Med. 2009;15(11):1318-1321.

11. Tobin MK, et al. Neurogenesis and inflammation after ischemic stroke: what is known and where we go from here. JCereb Blood Flow Metab. 2014;34(10):1573-1584.

12. $\mathrm{Fu} \mathrm{Y}$, et al. Immune interventions in stroke. Nat Rev Neurol. 2015;11(9):524-535.

13. Koraka P, et al. Plasma levels of inter-alpha inhibitor proteins in children with acute Dengue virus infection. PLoS One. 2010;5(4):e9967.

14. Wu R, et al. Delayed administration of human inter-alpha inhibitor proteins reduces mortality in sepsis. Crit Care Med. 2004;32(8):1747-1752.

15. Zhuo L, et al. Inter-alpha-trypsin inhibitor, a covalent protein-glycosaminoglycan-protein complex. J Biol Chem. 2004;279(37):38079-38082.

16. Chaaban $\mathrm{H}$, et al. Inter- $\alpha$ inhibitor protein and its associated glycosaminoglycans protect against histone-induced injury. Blood. 2015;125(14):2286-2296.

17. Singh K, et al. Inter-alpha inhibitor protein administration improves survival from neonatal sepsis in mice. Pediatr Res. 2010;68(3):242-247.

18. Disdier C, et al. Alterations in inter-alpha inhibitor protein expression after hypoxic-ischemic brain injury in neonatal rats. Int J Dev Neurosci. 2018;65:54-60.

19. Fries E, Kaczmarczyk A. Inter-alpha-inhibitor, hyaluronan and inflammation. Acta Biochim Pol. 2003;50(3):735-742.

20. Delsesto D, Opal SM. Future perspectives on regulating pro-and anti-inflammatory responses in sepsis. Contrib Microbiol. 2011;17:137-156.

21 . Htwe SS, et al. Inter- $\alpha$ inhibitor proteins maintain neutrophils in a resting state by regulating shape and reducing ROS production. Blood $A d v$. 2018;2(15):1923-1934.

22. Garantziotis S, et al. Inter-alpha-trypsin inhibitor attenuates complement activation and complement-induced lung injury. J Immunol. 2007;179(6):4187-4192.

23. Shah BA, et al. Blood level of inter-alpha inhibitor proteins distinguishes necrotizing enterocolitis from spontaneous intestinal perforation. J Pediatr. 2017;180:135-140.

24. Chaaban $\mathrm{H}$, et al. Inter-alpha inhibitor protein level in neonates predicts necrotizing enterocolitis. J Pediatr. 2010;157(5):757-761.

25. Yamashiro K, et al. Gut dysbiosis is associated with metabolism and systemic inflammation in patients with ischemic stroke. PLoS One. 2017;12(2):e0171521.

26. Spasova MS, et al. Ischemia reduces inter-alpha inhibitor proteins in the brain of the ovine fetus. Dev Neurobiol. 2017;77(6):726-737.

27. Yano T, et al. Neuroprotective effect of urinary trypsin inhibitor against focal cerebral ischemia-reperfusion injury in rats. Anesthesiology. 2003;98(2):465-473.

28. Abe $\mathrm{H}$, et al. [Effect of ulinastatin on delayed neuronal death in the gerbil hippocampus]. Masui. 1996;45(1):38-43.

29. Sjoberg EM, et al. Plasma clearance of rat bikunin: evidence for receptor-mediated uptake. Biochem J. 1995;308(pt 3):881-887.

30. Chen X, et al. Pharmacokinetics of inter-alpha inhibitor proteins and effects on hemostasis afte hypoxic-ischemic brain injury in neonatal rats. Curr Pharm Des. 2020;26(32):3997-4006.

31. Venna VR, et al. Inhibition of glycogen synthase kinase- $3 \beta$ enhances cognitive recovery after stroke: the role of TAK1. Learn Mem. 2015;22(7):336-343.

32. Barnes CA. Aging and the physiology of spatial memory. Neurobiol Aging. 1988;9(5-6):563-568.

33. Broadbent NJ, et al. Object recognition memory and the rodent hippocampus. Learn Mem. 2010;17(1):5-11.

34. Howe MD, et al. Fibronectin induces the perivascular deposition of cerebrospinal fluid-derived amyloid- $\beta$ in aging and after stroke. Neurobiol Aging. 2018;72:1-13.

35. Venna VR, et al. Chronic metformin treatment improves post-stroke angiogenesis and recovery after experimental stroke. Eur J Neurosci. 2014;39(12):2129-2138.

36. Spychala MS, et al. Age-related changes in the gut microbiota influence systemic inflammation and stroke outcome. Ann Neurol. 2018;84(1):23-36.

37. Chen Y, et al. A novel mouse model of thromboembolic stroke. J Neurosci Methods. 2015;256:203-211.

38. Liu Y, J et al. Standardizing a simpler, more sensitive and accurate tail bleeding assay in mice. World JExp Med. 2012;2(2):30-36.

39. Ritzel RM, et al. Aging alters the immunological response to ischemic stroke. Acta Neuropathol. 2018;136(1):89-110.

40. Cheng MH, et al. The outcomes of stroke induced by middle cerebral artery occlusion in different strains of mice. CNS Neurosci Ther. 2012;18(9):794-795.

41. Ren B, et al. Ulinastatin attenuates lung ischemia-reperfusion injury in rats by inhibiting tumor necrosis factor alpha. Transplant Proc. 2006;38(9):2777-2779.

42. Zhou LW, et al. Urinary trypsin inhibitor treatment ameliorates acute lung and liver injury resulting from sepsis in a rat model. Saudi Med J. 2008;29(3):368-373.

43. Inoue K, Takano H. Urinary trypsin inhibitor as a therapeutic option for endotoxin-related inflammatory disorders. Expert Opin Investig Drugs. 2010;19(4):513-520.

44. Shibata T, et al. Effects of protease inhibitors on postischemic recovery of the heart. Cardiovasc Drugs Ther. 1997;11(4):547-556.

45. Jonsson-Berling BM, Ohlsson K. Distribution and elimination of intravenously injected urinary trypsin inhibitor. Scand J Clin Lab Invest. 1991;51(6):549-557.

46. Karnad DR, et al. Intravenous administration of ulinastatin (human urinary trypsin inhibitor) in severe sepsis: a multicenter randomized controlled study. Intensive Care Med. 2014;40(6):830-838.

47. Linder A, Russell JA. An exciting candidate therapy for sepsis: ulinastatin, a urinary protease inhibitor. Intensive Care Med. 2014;40(8):1164-1167.

48. Jiang XM, et al. Effects of ulinastatin on global ischemia via brain pro-inflammation signal. Transl Neurosci. 2016;7(1):158-163.

49. Pemberton AD, et al. Proteomic identification of interactions between histones and plasma proteins: implications for cytoprotection. Proteomics. 2010;10(7):1484-1493.

50. Huang J, et al. DAMPs, ageing, and cancer: the 'DAMP Hypothesis'. Ageing Res Rev. 2015;24(pt A):3-16.

51. Chamorro A, et al. Neuroprotection in acute stroke: targeting excitotoxicity, oxidative and nitrosative stress, and inflammation. Lancet Neurol. 2016;15(8):869-881.

52. Ritzel RM, et al. Sex, stroke, and inflammation: the potential for estrogen-mediated immunoprotection in stroke. Horm Behav. 2013;63(2):238-253.

53. Ma Y, et al. Significance of complement system in ischemic stroke: a comprehensive review. Aging Dis. 2019;10(2):429-462.

54. Prame Kumar K, et al. Partners in crime: neutrophils and monocytes/macrophages in inflammation and disease. Cell Tissue Res. 2018;371(3):551-565.

55. Li X, et al. Chronic behavioral testing after focal ischemia in the mouse: functional recovery and the effects of gender. Exp Neurol. 2004;187(1):94-104.

56. Alawieh A, et al. Targeted complement inhibition salvages stressed neurons and inhibits neuroinflammation after stroke in mice. Sci Transl Med. 2018;10(441):eaao6459.

57. Sadler R, et al. Short-chain fatty acids improve poststroke recovery via immunological mechanisms. J Neurosci. 2020;40(5):1162-1173.

58. Carvelli J, et al. Association of COVID-19 inflammation with activation of the C5a-C5aR1 axis. Nature. 2020;588(7836):146-150.

59. Dick J, et al. C5a receptor 1 promotes autoimmunity, neutrophil dysfunction and injury in experimental anti-myeloperoxidase glomerulonephritis. Kidney Int . 2018;93(3):615-625.

60. Denk S, et al. Complement C5a-induced changes in neutrophil morphology during inflammation. Scand J Immunol. 2017;86(3):143-155.

61. Thundyil J, et al. C5a receptor (CD88) inhibition improves hypothermia-induced neuroprotection in an in vitro ischemic model. Neuromolecular Med. 2012;14(1):30-39.

62. Kim GH, et al. Protective effect of C5a receptor inhibition after murine reperfused stroke. Neurosurgery. 2008;63(1):122-125.

63. Clarke AR, et al. Therapeutic modulation of the complement cascade in stroke. Front Immunol. 2019;10:1723.

64. Biggins PJC, et al. The alternative receptor for complement component $5 \mathrm{a}, \mathrm{C} 5 \mathrm{aR} 2$, conveys 
neuroprotection in traumatic spinal cord injury. JNeurotrauma. 2017;34(12):2075-2085.

65. Roy-O'Reilly M, et al. CCL11 (Eotaxin-1) levels predict long-term functional outcomes in patients following ischemic stroke. Transl Stroke Res. 2017;8(6):578-584.

66. Logsdon AF, et al. Inter-alpha inhibitor proteins attenuate lipopolysaccharide-induced bloodbrain barrier disruption and downregulate circulating interleukin 6 in mice. J Cereb Blood Flow Metab. 2020;40(5):1090-1102.

67. Threlkeld SW, et al. Effects of inter-alpha inhibitor proteins on neonatal brain injury: age, task and treatment dependent neurobehavioral outcomes. Exp Neurol. 2014;261:424-433.

68. Venna VR, et al. Social interaction plays a critical role in neurogenesis and recovery after stroke. Transl Psychiatry. 2014;4:e351.

69. Venna VR, et al. NF- $\mathrm{KB}$ contributes to the detrimental effects of social isolation after experimental stroke. Acta Neuropathol. 2012;124(3):425-438.

70. Lee J, et al. Gut microbiota-derived short-chain fatty acids promote post-stroke recovery in aged mice. Circ Res. 2020;127(4):453-465.

71. Li J, et al. Effects of metformin in experimental stroke. Stroke. 2010;41(11):2645-2652.

72. Manwani B, et al. Functional recovery in aging mice after experimental stroke. Brain Behav Immun. 2011;25(8):1689-1700.

73. Li J, et al. Adenosine monophosphate activated protein kinase inhibition is protective in both sexes after experimental stroke. Neurosci Lett. 2010;482(1):62-65.

74. Motulsky HJ, Brown RE. Detecting outliers when fitting data with nonlinear regression - a new method based on robust nonlinear regression and the false discovery rate. BMC Bioinformatics. 2006;7:123. 